\title{
Equivalence and the Cartan Form
}

\author{
Peter J. Olver $\dagger$ \\ School of Mathematics \\ University of Minnesota \\ Minneapolis, Minnesota \\ U.S.A. $\quad 55455$
}

\begin{abstract}
An investigation into the Cartan form and nondegeneracy conditions for field-theoretic Lagrangians based on the Cartan equivalence method.
\end{abstract}

\section{Introduction.}

In the geometric theory of the calculus of variations in mechanics, the Cartan form, which first arose as the integrand in Hilbert's invariant integral, plays a ubiquitous role. One outstanding problem in the subject is how to define an appropriate Cartan form for a general variational problem. The case of higher order mechanics, i.e., variational problems in one independent variable, is also well understood, [25], [44]. Extensions to first order field theories, i.e., variational problems in more than one independent variable, are known, but here one is already confronted with several different possible Cartan forms, including Weyl's version, [51], the form proposed by Carathéodory, [10], and the more recent one proposed by Betounes, [7]. See [33], [43], for discussions. For higher order field theories, things are even less well understood, and there is considerable controversy over whether an appropriate Cartan form even exists. Two excellent surveys of the subject can be found in Kastrup, [32], which reviews the various field theories for multiple integrals, and Gotay, [22], which discusses the current literature on the subject.

Once one has settled on a Cartan form for a variational problem, the next issue is to determine suitable nondegeneracy conditions. In the geometric theory, the nondegeneracy condition usually arises from an attempt to define a suitable analogue of the classical Legendre transformation. Each different proposed Cartan form introduces different notions of nondegeneracy for variational problems, which has led to additional confusion in the literature. An alternative source of nondegeneracy conditions comes from the analysis of variational problems, most often via direct methods, and, in particular, the applications to linear and nonlinear elasticity. The key condition for first order variational

$\dagger$ Supported in Part by NSF Grant DMS 89-01600. 
problems is the well-known Legendre-Hadamard condition, $c f$. [37], for strong ellipticity. Surprisingly, for variational problems involving several independent and dependent variables, the geometrical nondegeneracy conditions are completely at odds with the analytical Legendre-Hadamard condition: obviously degenerate variational problems are often deemed nondegenerate by the geometric conditions of Weyl of Carathéodory, while simple strongly elliptic problems become degenerate; moreover, quadratic variational problems are not uniformly nondegenerate - the degeneracy in general varies from point to point. I will discuss in detail how the two kind of nondegeneracy conditions are related, how the Legendre-Hadamard condition can be deduced geometrically using some elementary representation theory of the general linear group, and argue that the general version of this condition, and not the Legendre transformation-based conditions, is the proper nondegeneracy condition for general variational problems.

It has been known for a long time that one of the first by-products of the Cartan equivalence method applied to the basic equivalence problem in the calculus of variations is the Cartan form, which already makes its appearance after the first set of reductions, [18], [28]. Thus, a natural approach to the determination of appropriate Cartan forms would be to begin the implementation of the equivalence method for general variational problems. The first attempt in this direction was the paper of Gardner and Shadwick, [19], which treated the case of first order scalar field theories, and recovered the usual Cartan form in this context. In her master's thesis, Bodnar, [8], treated the general first order variational problem, and made the important discovery that Carathéodory's Cartan form plays the key role. In this paper, we begin by reproducing this part of Bodnar's analysis, first because it has never appeared in the literature, and second, because she made an unfortunate error in her calculation of the non-degeneracy conditions arising from the second stage of the solution to the equivalence problem, which we rectify here. One of the useful consequences of the equivalence method is that it also determines the transformation properties of the relevant Cartan forms and nondegeneracy conditions. In particular, we deduce that Carathéodory's form is invariant under general point transformations (or even contact transformations in the case of a scalar theory), which is not the case with the Weyl form. Moreover, the derivation of the standard Legendre-Hadamard condition, or, alternatively, Carathéodory's nondegeneracy condition, proves that they too are invariant under point transformations, whereas the Weyl nondegeneracy condition is not.

We then proceed to higher order theories. In an earlier paper, [25], it was shown how the Cartan form of higher order mechanics can be found by formulating the equivalence problem for an $r^{\text {th }}$ order Lagrangian on the jet bundle $J^{k}$ for any $k \geq 2 r-1$. We will successfully derive an invariant Cartan form for a general second order Lagrangian through the equivalence method. However, for third and higher order variational problems, unexpected difficulties have thus far prevented us from determining a suitable Cartan form. The fact that significant new problems in the definition of Cartan forms arise for third and higher order field theoretic Lagrangians was first noted by Krupka, [34]; see also [1], [22]. Essentially we are unable to normalize crucial anti-symmetric components of a certain tensor appearing in a lifted version of the Cartan form through a direct analysis mimicking [25]. However, despite our failure to derive a suitable Cartan form for higher order Lagrangians, 
we are nevertheless able to determine the analogous nondegeneracy conditions. As with first order problems in several independent and dependent variables, the initial "Hessian tensor" governing the nondegeneracy includes some unusual skew-symmetric parts, which arise even in scalar field theories of order two or more. Representation-theoretic methods allow us to extract the more usual symmetric Hessian tensor, but even there we have more structure than one might at first suspect.

We begin by recalling the Cartan formulation of the standard equivalence problem for Lagrangians as presented in [30]. The notation used here is the same. We do not include divergence terms in our equivalence set-up, since the resulting equivalence problem is much more complicated. An interesting problem would be to implement a comparable part of the Cartan equivalence method for the divergence equivalence problem, but this appears to be extremely difficult.

\section{The Equivalence Problem.}

In order to establish a convenient notation, we begin with a very brief discussion of some basic facts in the theory of symmetric algebra of vector spaces as discussed, for example, in [16; chapter 1]. The symmetric algebra of a real (or complex) vector space $V$ is denoted by $\odot^{*} V=\oplus_{r>0} \odot^{r} V$; it can be identified with the space of all real-valued polynomial functions on the dual vector space $V^{*}$. If $\left\{e_{1}, \ldots, e_{p}\right\}$ is a basis of $V$, (which we can identify as linear functions on $V^{*}$ ), then $\odot^{r} V$ has induced basis $e_{I}=e_{i_{1}} \odot \cdots \odot e_{i_{r}}$, where $I$ runs over all symmetric multi-indices $I=\left(i_{1}, \ldots, i_{r}\right)$ with entries $1 \leq i_{\nu} \leq p$. Note that $e_{I}$ can be naturally viewed as a polynomial of degree $r$ on $V^{*}$. Given a symmetric multi-index $I$, we let $\widetilde{I}=\left(\widetilde{\imath}_{1}, \ldots, \widetilde{\imath}_{p}\right)$ denote the associated ordered multi-index, with each entry $\widetilde{\imath}_{k}$ denoting the numer of $k$ 's in $I$. For example, if $p=4$ and $I=(1,1,1,1,2,4,4,4)$, then $\widetilde{\imath}=(4,1,0,3)$. Note that $e_{I}=e^{\widetilde{I}}=\left(e_{1}\right)^{\tilde{\tau}_{1}} \odot \ldots \odot\left(e_{p}\right)^{\tilde{\imath}_{p}}$, where $\left(e_{j}\right)^{k}=e_{j} \odot \ldots \odot e_{j}$ is the $k^{\text {th }}$ symmetric power of $e_{j}$. Finally, define the factorial $I !=\widetilde{I} !=\widetilde{\imath}_{1} ! \widetilde{\imath}_{2} ! \cdots \widetilde{\imath}_{p}$ !

The general linear group GL( $V)$ acts naturally on the symmetric algebra $\odot^{*} V$. If $A=\left(A_{j}^{i}\right)$ is the matrix form of the linear transformation $A \in \mathrm{GL}(V)$ with respect to our basis $e_{i}$, then the corresponding $r^{\text {th }}$ symmetric power of $A$ has matrix form $\odot^{r} A=\left(A_{J}^{I}\right)$ with respect to the induced basis $e_{I}$ of $\bigodot^{r} V$, with entries

$$
A_{J}^{I}=\frac{1}{I !} \sum_{\pi} A_{j_{\pi(1)}}^{i_{1}} A_{j_{\pi(2)}}^{i_{2}} \ldots A_{j_{\pi(r)}}^{i_{r}}
$$

the sum being over all permutations $\pi$ of $\{1, \ldots, r\}$.

For a general variational problem, the underlying space (or bundle) is coordinatized by the independent variables $x \in \Omega \subset X=\mathbb{R}^{p}$, the dependent variables $u \in U=\mathbb{R}^{q}$, and their derivatives. (As all of our considerations are local, there is no loss in generality in formulating the problem over an open subdomain of Euclidean space. Moreover, the global, bundle-theoretic counterparts of our constructions are clear.) The fibers of the $r^{\text {th }}$ order jet space $J^{r}=J^{r}(X, U)$ are denoted by $U^{(r)}=U_{0} \oplus \cdots \oplus U_{r}$, where $U_{k}=\odot^{k} X^{*} \otimes U$. Local 
coordinates on $J^{r}$ are provided by the derivatives $u_{I}^{\alpha}=\partial^{k} u / \partial x^{I}$, indexed by $\alpha=1, \ldots, q$ and symmetric multi-indices $I=\left(i_{1}, \ldots, i_{k}\right)$, with $1 \leq i_{\nu} \leq p$, of order $k=\# I \leq r$. The contact ideal on $J^{r}, r \geq 1$, denoted $\mathcal{I}^{(r)}$, is generated by the contact forms

$$
\theta_{I}^{\alpha}=d u_{I}^{\alpha}-u_{I, i}^{\alpha} d x^{i}, \quad \alpha=1, \ldots, q, \quad 0 \leq \# I<r .
$$

Here, and in the sequel, lower case Latin letters run from 1 to $p$, lower case Greek letters from 1 to $q$, and upper case Latin letters are symmetric multi-indices. We will employ the summation convention on repeated upper and lower (multi-)indices throughout, although we will sometimes explicitly indicate the ranges of the summation indices. We further define the column vector of one-forms $\boldsymbol{\theta} \equiv\left(\boldsymbol{\theta}^{(0)}, \ldots \boldsymbol{\theta}^{(r-1)}\right)^{T}$, where $\boldsymbol{\theta}^{(k)}$ denotes the column vector of $k^{\text {th }}$ order contact forms $\theta_{I}^{\alpha}$, with $\# I=k$.

A local diffeomorphism $\Psi: J^{r} \rightarrow J^{r}$ defines a contact transformation if it preserves the contact ideal. This means that under the pull-back map on $T^{*} J^{r}$,

$$
\Psi^{*}(\overline{\boldsymbol{\theta}})=A \cdot \boldsymbol{\theta}
$$

for some (block lower triangular) matrix $A \in \mathrm{GL}\left(U^{(r)}\right)$. Bäcklund's Theorem, [4], [26], implies that any contact transformation is the prolongation of a point transformation $\Psi_{0}: J^{0} \rightarrow J^{0}$, or, in the case of a single dependent variable $(q=1)$, of a first order contact transformation $\Psi_{1}: J^{1} \rightarrow J^{1}$. (Interestingly, if one restricts to submanifolds of the jet space defined by system of differential equations, additional "internal" higher order contact transformations can exist; see [3] for a Bäcklund-style classification of these transformations.)

Consider a variational problem

$$
\mathcal{L}[u]=\int_{\Omega} L\left(x, u^{(r)}\right) d x
$$

with $r^{\text {th }}$ order Lagrangian $L$, where $d x=d x^{1} \wedge \cdots \wedge d x^{p}$ is the canonical volume form on $\Omega \subset X$. The standard equivalence problem for such an integral is to detemine when two such variational problems can be mapped to each other by a change of variables. Here we use the general pseudogroup of contact transformations, although restrictions to point transformations (which is unnecessary if $q>1$ ) or fiber-preserving transformations are readily treated using the same framework. (However, as with the simple one-dimensional problems, [28], one would not expect the Cartan form to naturally appear from the solution to the more restrictive fiber-preserving equivalence problem.) Thus, two $r^{\text {th }}$ order Lagrangians $L$ and $\bar{L}$ are said to be equivalent if there is a contact transformation $\Psi: J^{r} \rightarrow J^{r}$ such that the Lagrangian forms agreee under pull-back:

$$
\Psi^{*}(\bar{L} d \bar{x}) \equiv L d x \quad \bmod \mathcal{I}^{(r)}
$$

More generally, two Lagrangians are said to be divergence equivalent if they agree up to a divergence (null Lagrangian), so that

$$
\Psi^{*}(\bar{L} d \bar{x}) \equiv L d x+d \sigma \equiv(L+\operatorname{Div} P) d x \quad \bmod \mathcal{I}^{(r)},
$$


for some $(p-1)$-form $\sigma$, which, in local coordinates, is specified by a $p$-tuple of functions $P: J^{r} \rightarrow \mathbb{R}^{p}$. If two Lagrangians are divergence equivalent, then the associated EulerLagrange equations are mapped to each other by the equivalence. In [30] it was shown how to formulate both the standard and the divergence equivalence problems for $r^{\text {th }}$ order Lagrangians as Cartan equivalence problems on any jet bundle $J^{s}$ for $s \geq r$. However, in the analysis of higher order mechanics $(p=1, r>1)$, the Cartan form only appears directly if one formulates the problem on the jet bundle $J^{2 r-1}$, which is the minimal order jet bundle on which we can hope to construct a suitable Cartan form. See [25] for the derivation of the Cartan form for higher order mechanics, and [31] for an explanation of how the Cartan form manifests itself in terms of "derivative covariants" if one solves the Lagrangian equivalence problem on the minimal order jet bundle $J^{r}$.

We now review the Cartan formulation of the standard equivalence problem for a general $r^{\text {th }}$ order Lagrangian $L\left(x, u^{(r)}\right)$ in $p$ independent variables $x$, and $q$ dependent variables $u$. (The divergence equivalence problem has a similar, but more complicated formulation.) To begin with, we introduce a generally useful "barred" notation

$$
\bar{A}=A^{-1}, \quad \bar{p}=\frac{1}{p},
$$

for the inverses of a non-singular matrix $A$ and a nonzero scalar $p$. Assume for simplicity that we are on a domain where $L>0$. (If $L<0$, then we perform an orientation reversing transformation before beginning; the "singularities" where $L$ vanishes are not directly amenable to treatment by the Cartan approach, [28].) Our base coframe consists of the column vector of one-forms $\boldsymbol{\Omega}=(\widetilde{\boldsymbol{\theta}}, \boldsymbol{\omega}, \boldsymbol{\pi})^{T} \in T^{*} J^{r} \otimes J^{r}$, defined as follows. (In the notation $T^{*} J^{r} \otimes J^{r}$, the first tensor factor refers to the fact that each entry in $\Omega$ is a one-form on $J^{r}$, while the second refers to the fact that we can index the entries of $\Omega$ by the basis elements of the vector space $J^{r}$ relative to the given coordinate system. This notation, while convenent, is not coordinate-free.) The first set of coframe elements $\widetilde{\boldsymbol{\theta}}$ will be rescaled contact forms, which we assemble into a column vector

$$
\widetilde{\boldsymbol{\theta}}=\left(\boldsymbol{\theta}_{0}, L^{-\bar{p}} \boldsymbol{\theta}^{(1)}, L^{-2 \bar{p}} \boldsymbol{\theta}^{(2)}, \ldots, L^{-(r-1) \bar{p}} \boldsymbol{\theta}^{(r-1)}\right)^{T} \in T^{*} J^{r} \otimes U^{(r-1)} .
$$

The second set of coframe elements $\boldsymbol{\omega} \in T^{*} J^{r} \otimes X$ consists of the one-forms

$$
\omega^{i}=\sqrt[p]{L} d x^{i}=L^{\bar{p}} d x^{i}, \quad i=1, \ldots, p .
$$

Note that the wedge product of the forms $(2.8)$ is just the Lagrangian form: $\omega^{1} \wedge \cdots \wedge \omega^{p}=$ $L d x$. Finally, we append the column vector of one-forms $\pi \in T^{*} J^{r} \otimes U_{r}$, with entries

$$
\pi_{I}^{\alpha}=L^{-r \bar{p}} d u_{I}^{\alpha}, \quad \alpha=1, \ldots, q, \quad \# I=r .
$$

Vectors and matrices will be represented in block form relative to the components $\widetilde{\boldsymbol{\theta}}, \boldsymbol{\omega}, \boldsymbol{\pi}$ of the full coframe $\boldsymbol{\Omega}$. The structure group $G$ consists of matrices of the block form

$$
\left(\begin{array}{ccc}
A & 0 & 0 \\
B & J & 0 \\
C & D \cdot J & E
\end{array}\right)
$$


where $J \in \mathrm{SL}(X)$, (i.e., $\operatorname{det} J=1$ ), and $A \in \mathrm{GL}\left(U^{(2 r-2)}\right)$ is block lower triangular, with diagonal blocks $A_{k}=\bigodot^{k} J^{-T} \otimes A_{0}$ mapping $U_{k}=\odot^{k} X^{*} \otimes U$ to itself. We also have $B \in \operatorname{Hom}\left(U^{(2 r-2)}, X\right), C \in \operatorname{Hom}\left(U^{(2 r-2)}, U_{2 r-1}\right), D \in \operatorname{Hom}\left(X, U_{r}\right) \cap \odot^{k+1} X^{*} \otimes U$, where we identify $\operatorname{Hom}\left(X, U_{r}\right) \simeq X^{*} \otimes\left(\odot^{r} X^{*} \otimes U\right)$, and $E=\bigodot^{2 r-1} J^{-T} \otimes A_{0} \in \operatorname{GL}\left(U_{2 r-1}\right)$.

Theorem 1. Two Lagrangians $L$ and $\bar{L}$ are (standard) equivalent under a contact transformation if and only if there is a diffeomorphism $\Phi: J^{2 r-1} \rightarrow J^{2 r-1}$ which satisfies

$$
\Phi^{*}(\overline{\boldsymbol{\Omega}})=g \cdot \boldsymbol{\Omega}
$$

where $g$ is a $G$-valued function on $J^{2 r-1}$.

The implementation of the Cartan equivalence method, [11], [18], begins by introducing the "lifted coframe"

$$
\Gamma=g \cdot \Omega
$$

where $g$ is an arbitrary group element, so that $\boldsymbol{\Gamma}=(\boldsymbol{\vartheta}, \boldsymbol{\xi}, \boldsymbol{\pi})$ is a collection of one-forms on the principal bundle $J^{2 r-1} \times G$. In our case, the lifted coframe includes the one-forms

$$
\xi^{i}=J_{j}^{i} \varpi^{j}
$$

where

$$
\varpi^{j}=L^{\bar{p}} d x^{j}+\sum_{\# K=s \leq r-1} B_{\alpha}^{j, K} L^{-s \bar{p}} \theta_{K}^{\alpha}, \quad j=1, \ldots, p,
$$

which encode the transformation rules for the Lagrangian, and the one-forms

$$
\vartheta_{I}^{\alpha}=\sum_{\# L=s} A_{\beta}^{\alpha} \bar{J}_{I}^{K} L^{-s \bar{p}} \theta_{K}^{\beta}+\sum_{\# K=t<s} \widetilde{A}_{I, \beta}^{\alpha, K} L^{-t \bar{p}} \theta_{K}^{\beta}, \quad 0 \leq \# I=s<2 r-1
$$

which encode the fact that we are dealing with contact (or prolonged point) transformations. Here, in accordance with our convention (2.6), $\bar{J}=J^{-1}$, and $\bar{J}_{I}^{K}$ are the matrix entries of the symmetric power $\odot^{s} \bar{J}$, cf. (2.1). The group parameters $\widetilde{A}_{I, \beta}^{\alpha, K}$ are the offdiagonal block entries of the contact matrix $A$. Finally, note the important fact that the group parameters $B_{\alpha}^{j, K}$ are already symmetric under permutations of the multi-index $K$ (since $\theta_{K}^{\alpha}$ is symmetric in $K$ ), but are not automatically symmetric under permutations of the enlarged multi-index $(j, K)$. The coefficients $B_{\alpha}^{j, K}$ are of various orders; those of order $k$ are assembled into a subblock $B^{(k)} \equiv\left(B_{\alpha}^{j, K}\right), \# K=k-1$, so that $B=\left(B^{(1)}, \ldots, B^{(r)}\right)$. Bäcklund's Theorem implies that we can assume that the blocks in the submatrix $B$ will satisfy $B^{(k)}=0, k \geq 3$, when $q=1$, or $k \geq 2$, when $q>1$. However, as we remarked in [25], such an immediate restriction of the equivalence problem will not lead to the Cartan form for higher order problems since it will not include enough of the contact forms. Therefore, keeping the derivation in $[\mathbf{2 5}]$ in mind, the entire block matrix $B$ will be kept arbitrary for the time being. Note that there are also lifted coframe elements corresponding to the extra basis one-forms (2.9), but we will not need these in what is to follow. 
Using the coframe elements (2.14), we define the lifted Cartan form to be the $p$-form

$$
\Theta_{L}=\xi^{1} \wedge \xi^{2} \cdots \wedge \xi^{p}=\varpi^{1} \wedge \varpi^{2} \cdots \wedge \varpi^{p}
$$

the equality following from (2.13) and the fact that $J \in \mathrm{SL}(X)$. As it stands, $\Theta_{L}$ is a $p$-form on the principal bundle $J^{2 r-1} \times G$. Each time we reduce the structure group $G$, we will also be reducing the lifted Cartan form $\Theta_{L}$, with our ultimate goal being the determination of all the group parameters $B=\left(B^{(1)}, \ldots, B^{(r)}\right)$, as in the case of higher order mechanics discussed in [25], which will suffice to determine a Cartan form $\Theta_{c}$ which is independent of any group parameters. Note that, as in the first order case discussed in $[\mathbf{3 0}]$, we do not need to normalize the group parameters $J$, as they are unimodular to begin with, and therefore will not appear after we wedge the $\xi$ 's together as indicated in $(2.16)$.

\section{First Order Lagrangians.}

We begin by treating the first order case in detail, reproducing Bodnar's derivation of the Carathéodory form, [8], but then continuing on to find the nondegeneracy conditions related to the next batch of coframe normalizations coming from the Cartan equivalence method. Consider the standard equivalence problem for a first order variational problem

$$
\mathcal{L}[u]=\int_{\Omega} L\left(x, u^{(1)}\right) d x
$$

under the pseudogroup of contact transformations

$$
\bar{x}=\varphi\left(x, u^{(1)}\right), \quad \bar{u}=\psi\left(x, u^{(1)}\right), \quad \bar{u}_{i}^{\alpha}=\chi_{i}^{\alpha}\left(x, u^{(1)}\right) .
$$

(If $q>1$, then according to Bäcklund's Theorem, [26], the transformation necessarily reduces to a prolonged point transformation.) Using our general formulation of the standard Lagrangian equivalence problem, we can now apply the Cartan method to implement a solution. The lifted coframe $\boldsymbol{\Gamma}=(\boldsymbol{\vartheta}, \boldsymbol{\xi}, \boldsymbol{\pi})=g \cdot \boldsymbol{\Omega}, c f$. (2.12), consists of the one-forms

$$
\begin{aligned}
\vartheta^{\alpha} & =A_{\beta}^{\alpha} \theta^{\beta}, \\
\xi^{i} & =J_{j}^{i}\left(B_{\beta}^{j} \theta^{\beta}+L^{\bar{p}} d x^{j}\right), \\
\pi_{i}^{\alpha} & =C_{\beta, i}^{\alpha} \theta^{\beta}+D_{i j}^{\alpha} J_{k}^{j} L^{\bar{p}} d x^{k}+A_{\beta}^{\alpha} \bar{J}_{i}^{j} L^{-\bar{p}} d u_{j}^{\beta} .
\end{aligned}
$$

We now start the equivalence algorithm of absorption of torsion and normalization of group parameters. In the first loop through the algorithm, the only essential torsion terms are in the formula for $d \boldsymbol{\xi}$, which we compute, modulo the contact ideal, to be

$$
d \xi^{i} \equiv \varphi_{j}^{i} \wedge \xi^{j}+\tau^{i} \quad \bmod \mathcal{I}^{(0)}
$$


Here the $\varphi_{j}^{i}$ are the right-invariant (Maurer-Cartan) one-forms on the Lie group $G$ corresponding to the group parameters $J_{j}^{i}$. The torsion $\tau$ is given explicitly by

$$
\tau^{i}=Q_{\beta n}^{i m} \pi_{m}^{\beta} \wedge \xi^{n},
$$

in which

$$
Q_{\beta n}^{i m}=\bar{A}_{\beta}^{\alpha} J_{j}^{i} J_{k}^{m} \bar{J}_{n}^{l} P_{\alpha l}^{j k}, \quad P_{\alpha l}^{j k}=\bar{p} L^{\bar{p}-1} \frac{\partial L}{\partial u_{k}^{\alpha}} \delta_{l}^{j}-B_{\alpha}^{j} \delta_{l}^{k},
$$

where $\delta$ is the usual Kronecker delta, and we use the notation in (2.6). We note, for future reference, the trivial identities

$$
\delta_{i}^{i}=p, \quad J_{j}^{i} \bar{J}_{i}^{m}=\delta_{j}^{m} .
$$

(As in (2.6), $\bar{J}=J^{-1}$.) Now, since $J$ is restricted to have determinant 1 , the matrix of Maurer-Cartan forms $\left(\varphi_{j}^{i}\right)$ is trace-free, i.e., $\varphi_{i}^{i}=0$. Therefore, we can absorb all of the torsion coefficients $P$ except the trace

$$
Q_{\beta i}^{i m}=\bar{A}_{\beta}^{\alpha} J_{k}^{m} P_{\alpha j}^{j k},
$$

which constitutes essential torsion. The components (3.6) of the trace can be normalized to zero by requiring that $P$ have trace zero. which is achieved by normalizing the group parameters $B$ to be

$$
B_{\alpha}^{k}=L^{\bar{p}-1} \frac{\partial L}{\partial u_{k}^{\alpha}} .
$$

At this stage, we have already produced the Cartan form introduced by Carathéodory, [10]. More specifically, the normalizations (3.7) are the same as reducing the structure group to the subgroup $G_{1}$, found by setting $B=0$ in (2.10), (which happens to be the same as the structure group for the fiber-preserving equivalence problem), and replacing the original base coframe elements $\boldsymbol{\omega}$ by the new coframe elements

$$
\varpi^{i}=\sqrt[p]{L}\left\{d x^{i}+\frac{1}{L} \frac{\partial L}{\partial u_{i}^{\alpha}} \theta^{\alpha}\right\} .
$$

Since the group coefficients B have been normalized out, we immediately deduce that:

Proposition 2. Let $L$ and $\bar{L}$ be positive Lagrangians which are equivalent under the orientation-preserving contact transformation $\Psi: J^{1} \rightarrow J^{1}$, and let $\varpi$ and $\bar{\varpi}$ be the corresponding vectors of one-forms defined by (3.8). Then

$$
\Phi^{*}(\bar{\varpi})=J \varpi,
$$

where $J$ is a $p \times p$ matrix of functions on $J^{1}$ with $\operatorname{det} J=1$. In fact

$$
J=\frac{D \varphi}{\sqrt[p]{\operatorname{det} D \varphi}}
$$

where $D \varphi=\left(D_{j} \varphi^{i}\right)$ is the total Jacobian matrix of the base transformation $\bar{x}=\varphi(x, u)$. 


\section{Corollary 3. The Carathéodory $p$-form}

$$
\Theta_{C}=\varpi^{1} \wedge \ldots \wedge \varpi^{p}
$$

is invariant under general orientation-preserving contact transformations:

$$
\Psi^{*}\left(\bar{\Theta}_{C}\right)=\Theta_{C} .
$$

One of the crucial properties that any Cartan form must satisfy is that the EulerLagrange equations for the variational problem can be directly deduced therefrom, $[\mathbf{2 3}]$, $[\mathbf{2 2}]$, [43]. Specifically, suppose the $p$-form $\Theta$ is a Cartan form. We then require that if $\gamma: X \rightarrow J^{1}$ is any section of the first jet bundle, then $\gamma$ is the first prolongation of a solution to the Euler-Lagrange equations if and only if it satisfies

$$
\left.\gamma^{*}(\mathbf{v}\lrcorner d \Theta\right)=0,
$$

for every vertical vector field $\mathbf{v}$ on $J^{1}$. It is well known, $[\mathbf{2 2}]$, that condition (3.13) only serves to specify the terms of degree at most one in the contact forms. All the Cartan forms we deduce for higher order field theories will satisfy the suitable generalization of condition (3.13). Therefore, if (3.13) is the only constraint required of Cartan forms, it is relatively easy to construct global Cartan forms, although without extra structure (e.g., a connection) they will not be unique. Problems arise if one wishes that, in addition, the Cartan form inherit symmetries of the variational problem, leading to a global Noether Theorem, and it is here that the third and higher order cases offer additional as yet unresolved difficulties, [34].

Now, except in the special cases $p=1$ or $q=1$, the Carathéodory form depends nonlinearly the Lagrangian $L$. However, we have the expansion

$$
\Theta_{C}=\Theta_{W}+\Xi
$$

where the terms linear in $L$ are also of degree at most one in the contact forms,

$$
\Theta_{W}=L d x+\frac{\partial L}{\partial u_{i}^{\alpha}} \theta^{\alpha} \wedge \widehat{d x^{i}},
$$

where

$$
\left.\widehat{d x^{i}}=\frac{\partial}{\partial x^{i}}\right\lrcorner d x=(-1)^{i-1} d x^{1} \wedge \ldots \wedge d x^{i-1} \wedge d x^{i+1} \wedge \ldots \wedge d x^{p} .
$$

Also, the remaining terms $\Xi$ in (3.14) consist of wedge products of two or more contact forms $\theta^{\alpha}$. The $p$-form $\Theta_{W}$ defined by (3.15) is Weyl's candidate for a Cartan form for the variational problem, and (3.14) says that the Carathéodory form agrees with the Weyl form up terms of degree two or more in the contact forms. (In physics terminology, since $\Xi$ in (3.14) depends on inverse powers of the Lagrangian $L$, the Weyl form can be viewed as the "strong coupling limit" of the Carathéodory form, [32].) Therefore, as far as the Cartan formulation of the Euler-Lagrange equations goes, both the Carathéodory and the Weyl forms satisfy (3.13), and so are both viable candidates for a Cartan form. On the other hand, although quadratic and higher degree terms in the contact forms play no role in (3.13), such terms are essential if one wants to maintain invariance of the form under the full pseudogroup of point transformations. For $p>1, q>1$, the Weyl form is not invariant under a general point transformation! 
Example 4. Consider the case of a Lagrangian in 2 independent variables $x, y$ and one dependent variable $u$, so $p=2, q=1$. According to (3.11), (3.8), the Carathéodory Cartan form is given explicitly by

$$
\begin{aligned}
\Theta_{C} & =\left(\sqrt{L} d x-\frac{1}{\sqrt{L}} L_{u_{x}} \theta\right) \wedge\left(\sqrt{L} d y-\frac{1}{\sqrt{L}} L_{u_{y}} \theta\right) \\
& =L d x \wedge d y+\left(L_{u_{x}} d y-L_{u_{y}} d x\right) \wedge \theta,
\end{aligned}
$$

where $\theta=d u-u_{x} d x-u_{y} d y$ is the contact form. In this case, and, in fact, for general first order scalar field theories $(q=r=1)$, there are no non-zero higher order products of contact forms, and the Carathéodory form is the same as the Weyl form. Therefore, in this case the Weyl form also happens to be invariant under general contact transformations.

\section{Nondegeneracy Conditions.}

At this stage in our solution to the first order equivalence problem we are in a position to deduce nondegeneracy conditions for our Lagrangian. In the case of mechanics $(p=1)$ or scalar field theory $(q=1)$ there is not much controversy over what the appropriate conditions should be — namely the Hessian matrix of the Lagrangian with respect to the first order derivatives is required to be a non-singular symmetric matrix. Thus, for mechanics, the Hessian $\mathbf{H}=\left(\partial^{2} L / \partial u_{x}^{\alpha} \partial u_{x}^{\beta}\right)$ will be a symmetric $q \times q$ matrix, whereas for scalar field theory, $\mathbf{H}=\left(\partial^{2} L / \partial u_{i} \partial u_{j}\right), u_{i}=\partial u / \partial x^{i}$, will be a symmetric $p \times p$ matrix, each defining a quadratic form that plays a key role in the second variation of the integral. The transformation rules for the Lagrangian in both cases imply that the quadratic forms are transformed in a natural manner, and hence the algebraic characteristics (i.e., the rank, and, in the real case, the signature) are the complete invariants for the problem. More restrictively, classical convexity hypotheses on the Lagrangian, [20], or, what is essentially equivalent, ellipticity conditions on the Euler-Lagrange equations, require that the Hessian matrix be positive definite. In the case of field theory in several variables, $(p>1, q>1)$, matters are far less clear cut. First of all, the Hessian tensor $\mathbf{H}$ derived by the equivalence method no longer consists of just the second derivatives of the Lagrangian with respect to the first order derivatives $u_{i}^{\alpha}$ but, rather mysteriously, includes additional skew terms involving first order derivatives of $L$. Indeed, as we will find, the ordinary Hessian tensor is not invariant under arbitrary point transformations! Secondly, while the "Hessian tensor" $\mathbf{H}$, or even its (symmetrized) second order components, can be viewed as a symmetric matrix of size $(p q) \times(p q)$, the transformation rules for the Lagrangian only induce a restricted collection of transformation rules for the associated quadratic form; consequently, there are additional algebraic invariants beyond the rank and signature of H.

The geometric nondegeneracy conditions have essentially nothing to do with analytical conditions of ellipticity, such as the Legendre-Hadamard strong ellipticity condition, [37], which play such a crucial role in the applications to elasticity, not to mention the existence proofs based on direct methods. Indeed, the analysis points directly to the LegendreHadamard condition as crucial, so an important question is to determine how this condition 
is connected with the Hessian tensor constructed using the equivalence method. The Legendre-Hadamard condition no longer takes the form of requiring that a symmetric matrix be positive definite, but, rather, involves the positivity of a certain "biform". One of the main goals of this section is to see how these nondegeneracy conditions appear in the geometric framework of the equivalence approach. It turns out that this is quite pretty and relies on some elementary representation theory; the Hessian tensor naturally decomposes into a completely symmetric part and a "skew" part. The symmetric part of the Hessian tensor can be identified with the symmetric biform determining the Legendre-Hadamard condition; all the strange first order derivative terms are placed into the skew component and, I argue, should play no role in the nondegeneracy or degeneracy of the problem.

What constitutes an appropriate nondegeneracy condition for a variational problem? I would argue that, aside from connections with either analytical techniques or Legendre transformational/field theoretical concerns, any reasonable nondegeneracy condition should satisfy certain minimal requirements:

1. The condition must reduce to the standard nondegeneracy conditions in the case of mechanics $(p=1)$, or scalar field theory $(q=1)$.

2. The condition must be invariant under arbitrary point transformations (contact transformations in the case $q=1$ ).

3. The condition should only depend on the form of the Euler-Lagrange equations, not on the particular Lagrangian.

Analytical considerations would then further require that any strongly elliptic problem be automatically nondegenerate; moreover since a quadratic variational problem gives rise to a linear system of Euler-Lagrange equations, it would be good if its degeneracy or nondegeneracy depended only on the independent and dependent variables, not on the derivatives themselves. This means that the nondegeneracy condition should only depend on second order derivatives of the Lagrangian with respect to the derivatives of the field variables.

With this in mind, we now proceed to implement the next stage of the Cartan method. According to the normalizations (3.7), the reduced lifted coframe elements (2.14) take the form

$$
\xi^{i}=J_{j}^{i} \varpi^{j}=J_{j}^{i} L^{\bar{p}}\left\{d x^{j}+\frac{1}{L} \frac{\partial L}{\partial u_{j}^{\alpha}} \theta^{\alpha}\right\},
$$

cf. (3.8). We recompute the differential

$$
d \xi^{i}=\varphi_{j}^{i} \wedge \xi^{j}+\tau^{i},
$$

where the $\varphi_{j}^{i}$ are as before, but the $\tau^{i}$ are a new collection of torsion terms. In direct analogy with the analysis in the case of mechanics, [28], we should look at the $\boldsymbol{\pi} \wedge \boldsymbol{\vartheta}$ terms in the torsion $\boldsymbol{\tau}$, which have the form $Y_{\alpha \beta}^{i k} \pi_{k}^{\alpha} \wedge \vartheta^{\beta}$. The torsion coefficients $\mathbf{Y}=\left(Y_{\alpha \beta}^{i k}\right)$ are given by

$$
Y_{\alpha \beta}^{i k}=\bar{A}_{\alpha}^{\gamma} \bar{A}_{\beta}^{\delta} J_{j}^{i} J_{l}^{k} Z_{\gamma \delta}^{j l},
$$


where $\mathbf{Z}=\left(Z_{\alpha \beta}^{i k}\right)$ denotes the "augmented Hessian tensor", with components

$$
Z_{\alpha \beta}^{i k}=L^{2 \bar{p}-2}\left\{L \frac{\partial^{2} L}{\partial u_{i}^{\alpha} \partial u_{k}^{\beta}}+\frac{\partial L}{\partial u_{k}^{\alpha}} \frac{\partial L}{\partial u_{i}^{\beta}}-\frac{\partial L}{\partial u_{i}^{\alpha}} \frac{\partial L}{\partial u_{k}^{\beta}}\right\} .
$$

The first term in $\mathbf{Z}$ is, as expected, the Hessian matrix of $L$ with respect to the first order derivatives of $u$; however, the additional first order derivative terms are unusual, and unexpected. (In Bodnar's analysis, [8], the first derivatives terms in $\mathbf{Z}$ are omitted, due, I think, to an inadvertant computational error.) Note that if $i=k$, or if $\alpha=\beta$, then the first order derivative terms cancel out. Consequently, in the previously analyzed cases of mechanics $(p=1)$ and scalar field theory $(q=1)$ there are no first derivative terms in $\mathbf{Z}$, and hence, up to a power of of the Lagrangian, the augmented Hessian tensor $\mathbf{Z}$ agrees with the standard Hessian $\mathbf{H}$.

The next move in the Cartan procedure is to normalize the torsion tensor $\mathbf{Y}$ by suitable choice of the group parameters $A_{\beta}^{\alpha}, J_{j}^{i}$, a process which depends on the algebraic character of the augmented Hessian tensor $\mathbf{Z}$. When $p=1$ or $q=1$, both $\mathbf{Z}$ and $\mathbf{Y}$ are symmetric matrices, and we are essentially normalizing a quadratic form on either $X=\mathbb{R}^{p}$ or $U=\mathbb{R}^{q}$, a problem which is well understood. In these cases, the invariants are the rank and signature of the usual Hessian matrix of $L$ with respect to the derivative variables. The nondegeneracy conditions for a Lagrangian are just the nondegeneracy conditions that its Hessian matrix be nonsingular, and the analysis is reasonably straightforward, [18], [19].

Here we concentrate on cases when both $p$ and $q$ are greater than 1 , where far less is known. We begin by explaining the tensorial character of $\mathbf{Z}$ and $\mathbf{Y}$. Since they are both unchanged under simultaneous interchange of the pairs of indices $(j \alpha)$ and $(k, \beta)$, both of these tensors can be regarded as defining quadratic forms (symmetric matrices) on the tensor space $X^{*} \otimes U$, and hence can be regarded as elements of the second symmetric power $\odot^{2}\left(X \otimes U^{*}\right)$ of its dual. There is a natural action of the product group $\operatorname{GL}(X) \times \operatorname{GL}(U)$ on $\bigodot^{2}\left(X \otimes U^{*}\right)$, and, according to $(4.2)$,

$$
\mathbf{Y}=\left(J^{T} \otimes \bar{A}\right)^{T} \mathbf{Z}\left(J^{T} \otimes \bar{A}\right) .
$$

Thus, given $\mathbf{Z}$, the goal is to choose $J \in \mathrm{SL}(X), \bar{A} \in \mathrm{GL}(U)$ so as to put $\mathbf{Z}$ into canonical form.

As a direct consequence of the invariance of the Cartan procedure, we deduce that the augmented Hessian tensors of two Lagrangians must correspond:

Proposition 5. Let $L$ and $\bar{L}$ be equivalent Lagrangians under $\Phi: J^{1} \rightarrow J^{1}$, and let $\mathbf{Z}$ and $\overline{\mathbf{Z}}$ be the corresponding augmented Hessian tensors. Then

$$
\Phi^{*}(\overline{\mathbf{Z}})=\left(J^{T} \otimes \bar{A}\right)^{T} \mathbf{Z}\left(J^{T} \otimes \bar{A}\right),
$$

where $J$ is given by (3.10), and $\bar{A}=A^{-1}$, is the inverse of the $q \times q$ matrix $A$ with entries

$$
A_{\beta}^{\alpha}=\frac{\partial \psi^{\alpha}}{\partial u^{\beta}}-\chi_{i}^{\alpha} \frac{\partial \varphi^{i}}{\partial u^{\beta}}
$$


The standard Hessian tensor $\mathbf{H}=\left(H_{\alpha \beta}^{i k}\right)$ associated with a first order Lagrangian $L$ is given by

$$
H_{\alpha \beta}^{i k}=\frac{\partial^{2} L}{\partial u_{i}^{\alpha} \partial u_{k}^{\beta}} .
$$

If $p=1$ or $q=1$ then, up to a factor of $L$, the augmented Hessian coincides with the standard version, $\mathbf{Z}=L^{2 \bar{p}-1} \mathbf{H}$. Therefore, in these two cases, the ordinary Hessian is, up to a scalar factor, also invariant under general point (contact) transformations. However, when both $p, q>1$, the ordinary Hessian $\mathbf{H}$ is not generally invariant, even up to a factor, under arbitrary point transformations! Indeed, let $\bar{x}=\varphi(x, u), \bar{u}=\psi(x, u)$ be any point transformation, with first prolongation (3.2). Define $J=D \varphi$ to be the total Jacobian matrix (note that we are, temporarily, omitting the scalar determinantal factor from our earlier formula (3.10)) and let $A$ be defined by (4.6). Note the important identity

$$
\frac{\partial(\operatorname{det} J)}{\partial u_{i}^{\alpha}}=\frac{\partial \varphi^{k}}{\partial u^{\alpha}} \bar{J}_{k}^{i} \operatorname{det} J
$$

which follows from the fact that only the $i^{\text {th }}$ row of the matrix $J$ depends, affinely, on $u_{i}^{\alpha}$. Now, suppose

$$
\bar{L}\left(\bar{x}, \bar{u}^{(1)}\right) \operatorname{det} J=L\left(x, u^{(1)}\right)
$$

are equivalent Lagrangians. A fairly straightforward chain rule computation using (4.8) leads to the transformation formulas for first and second order derivatives of the Lagrangians:

$$
\frac{\partial L}{\partial u_{i}^{\alpha}}=\bar{J}_{l}^{i}\left(A_{\alpha}^{\gamma} \frac{\partial \bar{L}}{\partial \bar{u}_{l}^{\gamma}}+\bar{L} \frac{\partial \varphi^{l}}{\partial u^{\alpha}}\right) \operatorname{det} J
$$

and

$$
\frac{\partial^{2} L}{\partial u_{i}^{\alpha} \partial u_{j}^{\beta}}=\bar{J}_{l}^{i} \bar{J}_{m}^{j}\left\{A_{\alpha}^{\gamma} A_{\beta}^{\delta} \frac{\partial^{2} \bar{L}}{\partial \bar{u}_{l}^{\gamma} \partial \bar{u}_{m}^{\delta}} W_{\alpha \beta}^{l m}\right\} \operatorname{det} J,
$$

where the additional "skew" terms, which prohibit the invariance of the Hessian, are

$$
W_{\alpha \beta}^{l m}=A_{\alpha}^{\gamma} \frac{\partial \varphi^{m}}{\partial u^{\beta}} \frac{\partial \bar{L}}{\partial \bar{u}_{l}^{\gamma}}-A_{\alpha}^{\gamma} \frac{\partial \varphi^{l}}{\partial u^{\beta}} \frac{\partial \bar{L}}{\partial \bar{u}_{m}^{\gamma}}+A_{\beta}^{\gamma} \frac{\partial \varphi^{m}}{\partial u^{\alpha}} \frac{\partial \bar{L}}{\partial \bar{u}_{l}^{\gamma}}-A_{\beta}^{\gamma} \frac{\partial \varphi^{m}}{\partial u^{\alpha}} \frac{\partial \bar{L}}{\partial \bar{u}_{m}^{\gamma}} .
$$

Note that if $l=m$ or $\alpha=\beta$, then $W_{\alpha \beta}^{l m}=0$. Thus, in the case of mechanics $(p=1)$ or scalar field theory $(q=1)$, the tensor $\mathbf{W}$ is identically zero, which provides a direct proof of the invariance of the Hessian in these two cases. However, in general $\mathbf{W} \neq 0$, and the (standard) Hessian is not invariant, although the reader can readily use (4.9), (4.10), (4.11) to demonstrate the invariance of the augmented Hessian (4.3).

In the Carathéodory theory, the existence of a suitable Legendre transformation requires the invertibility of the symmetric $(p q) \times(p q)$ matrix corresponding to the tensor $\mathbf{Z}$. In view of (4.5), the consequential nondegeneracy condition is clearly unaffected by general contact transformations. 


\section{Proposition 6. The Carathéodory nondegeneracy condition}

$$
\operatorname{det} \mathbf{Z} \neq 0
$$

is invariant under arbitrary contact transformations.

On the other hand, the Weyl nondegeneracy condition requires that the $(p q) \times(p q)$ matrix determined corresponding to the ordinary Hessian tensor $\mathbf{H}$ is nonsingular:

$$
\operatorname{det} \mathbf{H} \neq 0 \text {, }
$$

and is not invariant under general point transformations, unless either $p=1$ or $q=1$. Thus, nondegeneracy in the Weyl sense depends on the particular coordinate system in use, and thus does not satisfy one of our basic requirements. The Carathédory condition does satisfy this invariance criterion, but, as I shall argue, fails to be appropriate for other reasons.

We now connect the geometrically derived (augmented) Hessian tensor with the analytical Legendre-Hadamard condition. The key observation comes from the representation theory of the general linear group (or, equivalently, the theory of symmetry classes of tensors - see [51] and Section 5 below for more details). If neither $X$ nor $U$ is one-dimensional, then the space $\odot^{2}\left(X \otimes U^{*}\right)$ is not an irreducible representation module for the action of the product group $\mathrm{GL}(X) \times \mathrm{GL}(U)$, but decomposes into two irreducible summands. It is this decomposition that provides the crucial connection between the geometric approach and the analytical ellipticity conditions of Legendre and Hadamard.

Lemma 7. There is a canonical vector space decomposition

$$
\odot^{2}\left(X \otimes U^{*}\right)=\left(\odot^{2} X \otimes \odot^{2} U^{*}\right) \oplus\left(\bigwedge^{2} X \otimes \bigwedge^{2} U^{*}\right)
$$

which is preserved under the action (4.4) of $\mathrm{GL}(X) \times \mathrm{GL}(U)$.

Let $\pi_{\odot}: \odot^{2}\left(X \otimes U^{*}\right) \rightarrow \odot^{2} X \otimes \odot^{2} U^{*}$ and $\pi_{\wedge}: \odot^{2}\left(X \otimes U^{*}\right) \rightarrow \Lambda^{2} X \otimes \Lambda^{2} U^{*}$ denote the projections based on (4.14). Given $\mathbf{Z} \in \odot^{2}\left(X \otimes U^{*}\right)$, we write $\mathbf{S}=\pi_{\odot}(\mathbf{Z})$ for the component of $\mathbf{Z}$ in $\odot^{2} X \otimes \odot^{2} U^{*}$, and $\mathbf{W}=\pi_{\wedge}(\mathbf{Z})$ for the component of $\mathbf{Z}$ in $\wedge^{2} X \otimes \wedge^{2} U^{*}$, so that $\mathbf{Z}=\mathbf{S}+\mathbf{W}$, where

$$
S_{\alpha \beta}^{i k}=\frac{1}{2}\left\{Z_{\alpha \beta}^{i k}+Z_{\alpha \beta}^{k i}\right\}, \quad W_{\alpha \beta}^{i k}=\frac{1}{2}\left\{Z_{\alpha \beta}^{i k}-Z_{\alpha \beta}^{k i}\right\} .
$$

Note that $\mathbf{S}$ is invariant under the interchange of either $i$ and $k$ or of $\alpha$ and $\beta$, so we will call $\mathbf{S}$ the symmetric component of the tensor $\mathbf{Z}$; similarly, $\mathbf{W}$ changes sign if we interchange either $i$ and $k$, or $\alpha$ and $\beta$ (but is symmetric under a simultaneous interchange of both pairs of indices), and will be called the skew component. Note that, by construction, the action of $\mathrm{GL}(X) \times \mathrm{GL}(U)$ on $\odot^{2} X \otimes \odot^{2} U^{*}$ given by (4.4) preserves the splitting of such tensors into symmetric and skew components. 
For our Lagrangian, according to (4.15), the symmetric component $\mathbf{S}=\pi_{\odot}(\mathbf{Z})$ of the augmented Hessian tensor (4.3) is given by $\mathbf{S}=L^{2 \bar{p}-2} \boldsymbol{\Sigma}$, where $\boldsymbol{\Sigma}=\pi_{\odot}(\mathbf{H})$ is the symmetric component of the standard Hessian (4.7), with coefficients

$$
\Sigma_{\alpha \beta}^{i k}=\frac{1}{2}\left\{\frac{\partial^{2} L}{\partial u_{i}^{\alpha} \partial u_{k}^{\beta}}+\frac{\partial^{2} L}{\partial u_{k}^{\alpha} \partial u_{i}^{\beta}}\right\} .
$$

The skew component of the augmented Hessian is the tensor $\mathbf{W}=\pi_{\wedge}(\mathbf{Z})$ with coefficients

$$
W_{\alpha \beta}^{i k}=L^{2 \bar{p}-1}\left\{\frac{1}{2} L\left(\frac{\partial^{2} L}{\partial u_{i}^{\alpha} \partial u_{k}^{\beta}}-\frac{\partial^{2} L}{\partial u_{k}^{\alpha} \partial u_{i}^{\beta}}\right)+\left(\frac{\partial L}{\partial u_{k}^{\alpha}} \frac{\partial L}{\partial u_{j}^{\beta}}-\frac{\partial L}{\partial u_{j}^{\alpha}} \frac{\partial L}{\partial u_{k}^{\beta}}\right)\right\}
$$

which is rather unusual. Despite its invariance, this tensor apparently plays no direct role in the analysis of variational problems.

As a consequence of Lemma 7 , if $L$ and $\bar{L}$ are equivalent Lagrangians, then their symmetric Hessian tensors $\mathbf{S}$ and $\overline{\mathbf{S}}$ (and also their skew Hessian tensors $\mathbf{W}$ and $\overline{\mathbf{W}}$ ) satisfy the same invariance condition (4.5) as the augmented Hessians; consequently, whereas the ordinary Hessian is not invariant, its symmetrized counterpart is invariant up to a multiplicative factor.

The symmetric Hessian can also be derived by restricting the augmented Hessian, which is a quadratic form on $X \otimes U^{*}$, to the subset of rank one tensors. Recall that a tensor $\eta \in X \otimes U^{*}$ has rank one if and only if it is of the form $\eta=\xi \otimes \lambda$ for $\xi \in X$, $\lambda \in U^{*}$, and so has components $\eta_{i}^{\alpha}=\xi_{i} \lambda^{\alpha}$. Evaluating $\mathbf{Z}$ or $\mathbf{S}$ on this subset results in the biquadratic biform

$$
S[\xi, \lambda]=\mathbf{S}[\xi \otimes \lambda]=\mathbf{Z}[\xi \otimes \lambda]=S_{\alpha \beta}^{i k} \xi_{i} \xi_{k} \lambda^{\alpha} \lambda^{\beta}=L^{2 \bar{p}-2}\left\{\frac{\partial^{2} L}{\partial u_{i}^{\alpha} \partial u_{k}^{\beta}} \xi_{i} \xi_{k} \lambda^{\alpha} \lambda^{\beta}\right\} .
$$

(By definition, a biform of bidegree $(m, n)$ is a function $P(\xi, \lambda)$ of two sets of variables $\xi \in \mathbb{R}^{p}, \lambda \in \mathbb{R}^{q}$, which, for each fixed $\lambda$ is a homogeneous polynomial of degree $m$ in $\xi$, and, for each fixed $\xi$ is a homogeneous polynomial of degree $n$ in $\lambda$. In our case, the bidegree is $(2,2)$. See $[\mathbf{4 7}],[\mathbf{4 8}]$, for remarks and references.) Note that the skew component of the augmented Hessian vanishes when restricted to the space of rank one tensors. The biquadratic biform (4.18) transforms in the obvious manner under the product group $\mathrm{GL}(X) \times \mathrm{GL}(U)$, and so its classical invariants and covariants will play a key role in the analysis of nondegeneracy conditions and canonical forms. Unfortunately, however, the invariant theory of biforms is not well understood except in the case $p=q=2,[\mathbf{4 9}],[\mathbf{4 0}]$; see also the remarks below.

The symbol of the variational problem with Lagrangian $L$ is the biform obtained from $S[\xi, \lambda]$ by omitting the power of $L$, or, equivalently, by evaluating the ordinary Hessian (4.7) on the space of rank one tensors: $\Sigma[\xi, \lambda]=L^{2-2 \bar{p}} S[\xi, \lambda]=\mathbf{H}[\xi \otimes \lambda]$; explicitly,

$$
\Sigma[\xi, \lambda]=\sum_{i, k, \alpha, \beta} \frac{\partial^{2} L}{\partial u_{i}^{\alpha} \partial u_{k}^{\beta}} \xi_{i} \xi_{k} \lambda^{\alpha} \lambda^{\beta}
$$


Note that, under point (contact) transformations, the symbol $\Sigma[\xi, \lambda]$ is conformally invariant, meaning invariant up to a multiplicative factor. The Legendre-Hadamard strong ellipticity condition, [6], [24], [37], requires that the symbol be positive definite, meaning that

$$
\Sigma[\xi, \lambda]>0 \quad \text { for all } \quad 0 \neq \xi \in X, \quad 0 \neq \lambda \in U^{*} .
$$

Condition (4.20) results from imposing a positivity condition on the second variation of the variational problem, and (provided $L$ is at least $C^{2}$ ) is equivalent to rank one convexity, [5]. The conformal invariance of the symbol implies:

Theorem 8. The Legendre-Hadamard strong ellipticity condition (4.19), (4.20), is invariant under arbitrary point (contact) transformations.

In light of these considerations, we propose that the proper nondegeneracy condition for a multivariable first order Lagrangian is that the symmetric Hessian biform $S[\xi, \lambda]$ (or, equivalently, the symbol $\Sigma[\xi, \lambda]$ ) be nondegenerate in the following sense. Recall first that a quadratic form $Q[\xi]=\xi^{T} A \xi$ is nondegenerate if $\operatorname{det} A \neq 0$. An equivalent formulation of this condition is to consider the associated symmetric bilinear form $\widehat{Q}[\xi, \eta]=\xi^{T} A \eta$, obtained by polarization, and require that, for each $\eta \neq 0$, the linear function $L_{\eta}[\xi]=$ $\widehat{Q}[\xi, \eta]$ is nonzero. Let

$$
B[\xi, \lambda]=(\xi \otimes \lambda)^{T} \mathbf{C}(\xi \otimes \lambda)=C_{\alpha \beta}^{i k} \xi_{i} \xi_{k} \lambda^{\alpha} \lambda^{\beta}
$$

be a general biform where, without loss of generality, the coefficient "matrix" $\mathbf{C}=\left(C_{\alpha \beta}^{i k}\right)$ is symmetric, meaning $C_{\alpha \beta}^{i k}=C_{\beta \alpha}^{k i}$. We define the polarization of $B$ to be the "bi-bilinear form"

$$
\widehat{B}[\xi, \eta ; \lambda, \mu]=(\xi \otimes \lambda)^{T} \mathbf{C}(y \otimes v)=C_{\alpha \beta}^{i k} \xi_{i} \eta_{k} \lambda^{\alpha} \mu^{\beta} .
$$

Then the biquadratic biform $B$ is called nondegenerate if and only if for each $\eta \neq 0, \mu \neq 0$, the bilinear function $L_{\eta, \mu}[\xi, \eta]=\widehat{B}[\xi, \eta ; \lambda, \mu]$ is nondegenerate (i.e., not identically zero). Clearly any positive definite biform is nondegenerate. This definition and its consequences will be a topic for subsequent research.

Consider the special case of quadratic variational problems, whose Euler-Lagrange equations are linear. (Such variational problems are basic to the study of linear elasticity.) In general, a quadratic Lagrangian is determined by a tensor $\mathbf{C} \in \odot^{2}\left(X^{*} \otimes U\right)$, so that

$$
L_{\mathbf{C}}(\nabla u)=C_{\alpha \beta}^{i k} u_{i}^{\alpha} u_{k}^{\beta}
$$

If we break $\mathbf{C}=\mathbf{S}+\mathbf{W}$ up into its symmetric $\mathbf{S}=\pi_{\odot}(\mathbf{C})$ and skew $\mathbf{W}=\pi_{\wedge}(\mathbf{C})$ components, $c f$. (4.15) with $\mathbf{C}$ replacing $\mathbf{H}$, this induces a decomposition of the quadratic Lagrangian

$$
L_{\mathbf{C}}=L_{\mathbf{S}}+L_{\mathbf{W}}
$$

The key remark is that the skew component $L_{\mathbf{W}}$ is a null Lagrangian or total divergence, [6]. Therefore, two quadratic Lagrangians differ by a divergence if and only if they have 
the same symmetric components, and so one can use the symmetric component $L_{\mathbf{S}}$ is a canonical representative of the quadratic Lagrangian $L$. Consequently, the symmetric Hessian tensors are in one-to-one correspondence with the set of conservative (meaning Euler-Lagrange) systems of second order linear partial differential equations. Indeed, the classification of quadratic Lagrangians for $p=q=2$ done in [40] was essentially a classification of the symmetric tensors $\mathbf{S} \in \bigodot^{2} X^{*} \otimes \bigodot^{2} U$. Moreover, once we have canonical forms for these tensors, it is simple to get canonical forms for the more general tensors $\mathbf{C} \in \odot^{2}\left(X^{*} \otimes U\right)$ - one just normalizes its symmetric component, and, in the degenerate cases, uses any residual symmetry of the tensor to try to further normalize the skew component. (This is the same as the problem of determining canonical forms for the top order terms, $C_{\alpha \beta}^{i k} u_{i k}^{\beta}$, of general systems of $q$ linear, second order, constant coefficient partial differential equations in $q$ unknowns.)

The invariance of the symmetric Hessian tensor under the more comprehensive notion of divergence equivalence holds in full generality.

Proposition 9. Let $L$ and $\bar{L}$ be divergence equivalent Lagrangians under the point (contact) transformation $\Phi: J^{1} \rightarrow J^{1}$. Let $\mathbf{S}$ and $\overline{\mathbf{S}}$ be the corresponding symmetric Hessian tensors, $c f .(4.16)$. Then

$$
\Phi^{*}(\overline{\mathbf{S}})=\left(J^{T} \otimes \bar{A}\right)^{T} \mathbf{S}\left(J^{T} \otimes \bar{A}\right),
$$

where $J$ is given by (3.10), $\bar{A}=A^{-1}$, where $A$ is given by (4.6).

\section{Proof.}

In view of Proposition 5 , it suffices to prove that if $L$ and $\bar{L}$ differ by a null Lagrangian $N$, then $\overline{\mathbf{S}}=\mathbf{S}$. Recall, [38], that every first order null Lagrangian has the form

$$
N\left(x, u^{(1)}\right)=\sum_{A, K} \rho_{K}^{A}(x, u) N_{K}^{A}(\nabla u)
$$

where the $N_{K}^{A}$ are Jacobian determinants

$$
N_{K}^{A}=\frac{\partial\left(u^{\alpha_{1}}, \ldots, u^{\alpha_{m}}\right)}{\partial\left(x^{k_{1}}, \ldots, x^{k_{m}}\right)}
$$

indexed by pairs of increasing multi-indices $K=\left(k_{1}<k_{2}<\cdots<k_{m}\right), 1 \leq k_{\nu} \leq p$, and $A=\left(\alpha_{1}<\alpha_{2}<\cdots<\alpha_{m}\right), 1 \leq \alpha_{\mu} \leq q$, and $m$ ranges between 1 and $p$. The coefficients

$\rho_{K}^{A}$ depend only on $x$ and $u$, so each null Lagrangian depends on the first order derivatives of $u$ in a purely skew fashion, and a simple calculation proves that the symmetric Hessian of each $N_{K}^{A}$ is zero.

Q.E.D.

The skew Hessian is certainly not zero for general null Lagrangians (4.23). Since both the Weyl and Carathéodory nondegeneracy conditions include the skew Hessian, neither is invariant under the more general notion of divergence equivalence. Moreover, the Carathéodory condition does not even uniquely characterize nondegeneracy for quadratic 
Lagrangians, since the determinant (4.12) depends on the values of $\nabla u$, and so vanishes on subsets of $J^{1}$, even in the case of strongly elliptic quadratic Lagrangians. These considerations will become clearer if we consider a couple of simple examples, one extremely degenerate, and one strongly elliptic.

Example 10. Suppose $p=2, q=1$. Consider the quadratic Lagrangian

$$
L=u_{x}^{2}+\gamma\left(u_{x} v_{y}-u_{y} v_{x}\right)
$$

where $\gamma$ is a constant. The term in parentheses constitutes the unique quadratic null Lagrangian in this context, so the Euler-Lagrange equations for $L$ are merely

$$
u_{x x}=0, \quad 0=0,
$$

independent of $\gamma$. Consequently, any reasonable person would, I think, not hesitate in calling this Lagrangian degenerate. Nevertheless, when $\gamma \neq 0$, the Lagrangian $L$ passes both the Weyl and the Carathéodory nondegeneracy tests (the latter provided $u_{x} \neq 0$ )! Restricting to the domain where $L>0$, we define the functions

$$
R_{C}=-\frac{\gamma u_{x}^{2}}{L}, \quad R_{W}=\gamma
$$

the subscripts standing for "Carathéodory" and "Weyl" respectively. Consider the $4 \times 4$ matrices $M_{C}, M_{W}$, each of the form

$$
M=\left(\begin{array}{cccc}
2 & 0 & 0 & R \\
0 & 0 & -R & 0 \\
0 & -R & 0 & 0 \\
R & 0 & 0 & 0
\end{array}\right),
$$

where $R$ is either $R_{C}$ or $R_{W}$. The Carathéodory nondegeneracy condition is that $M_{C}$ be nonsingular, which, for $\gamma \neq 0$, is satisfied provided $u_{x} \neq 0$. The Weyl nondegeneracy condition is that $M_{W}$, be nonsingular, and so, provided $\gamma \neq 0$ is always satisfied! This, at least in my opinion, is absurd, and motivates rethinking the entire nondegeneracy assumptions underlying both theories. On the other hand, the symbol of the Lagrangian (4.24) is the quadratic biform $\Sigma[\xi, \eta ; \lambda, \mu]=\xi^{2} \lambda^{2}$, which $i s$ degenerate according to the preceding defintion.

Example 11. An orthotropic Lagrangian is one of the special form

$$
L=u_{x}^{2}+\alpha u_{y}^{2}+2 \beta u_{x} v_{y}+\alpha v_{x}^{2}+v_{y}^{2} .
$$

The corresponding Euler-Lagrange equations are thus equivalent to the "orthotropic Navier equations"

$$
u_{x x}+\alpha u_{y y}+\beta v_{x y}=0, \quad \beta u_{x y}+\alpha v_{x x}+v_{y y}=0 .
$$

In the strongly elliptic case, the parameters $\alpha$ and $\beta$ represent two canonical elastic moduli. A basic result, [41], is that every strongly elliptic, constant coefficient, quadratic Lagrangian is, up to the addition of a null Lagrangian, equivalent to an orthotropic Lagrangian. 
Theorem 12. Let $L(\nabla u)$ be a homogeneous first order planar quadratic Lagrangian which satisfies the Legendre-Hadamard strong ellipticity condition. Then, under a linear change $x \mapsto A x, u \mapsto B u$ of independent variables and dependent variables, $L$ is divergence equivalent to a orthotropic Lagrangian (4.26), where the canonical elastic moduli $\alpha$ and $\beta$ satisfy the strong ellipticity inequalities $\alpha>0,|\beta|<\alpha+1$.

A more complete classification of canonical forms for such quadratic Lagrangians can be found in [40]. Beyond the trivial cases $p=1$ or $q=1$, this is the only case in which a complete list of canonical forms for quadratic Lagrangians is known, although a strongly elliptic canonical form in the case $p=2, q=3$, has recently been proposed, [42].

We consider a Lagrangian of the slightly more general form

$$
\widehat{L}=u_{x}^{2}+\alpha u_{y}^{2}+2 \beta u_{x} v_{y}+\alpha v_{x}^{2}+v_{y}^{2}+\gamma\left(u_{x} v_{y}-u_{y} v_{x}\right),
$$

differing from the orthotropic Lagrangian (4.26) by a null Lagrangian, and hence determining the same Euler-Lagrange equations (4.27). We compute the Cartan forms and nondegeneracy conditions for such Lagrangians. The Carathéodory form is the wedge product of the two one-forms

$$
\begin{aligned}
& \xi^{x}=\sqrt{L} d x+\frac{1}{\sqrt{L}}\left[\left(u_{x}+(\beta+\gamma) v_{y}\right) \theta^{u}+\left(\alpha v_{x}-\gamma u_{y}\right) \theta^{v}\right] \\
& \xi^{y}=\sqrt{L} d y+\frac{1}{\sqrt{L}}\left[\left(\alpha u_{y}-\gamma v_{x}\right) \theta^{u}+\left((\beta+\gamma) u_{x}+v_{y}\right) \theta^{v}\right],
\end{aligned}
$$

where $\theta^{u}=d u-u_{x} d x-u_{y} d y, \theta^{v}=d v-v_{x} d x-v_{y} d y$, and is given explicitly as

$$
\begin{aligned}
\Theta_{C}=L d x \wedge d y & +\left[\left(\gamma v_{x}-\alpha u_{y}\right) d x-\left(u_{x}+(\beta+\gamma) v_{y}\right) d y\right] \wedge \theta^{u} \\
& +\left[\left((\beta+\gamma) u_{x}+v_{y}\right) d x+\left(\alpha v_{x}-\gamma u_{y}\right) d y\right] \wedge \theta^{v}+R \theta^{u} \wedge \theta^{v},
\end{aligned}
$$

where

$$
R=\frac{(\beta+\gamma)\left(u_{x}^{2}+v_{y}^{2}\right)+\alpha \gamma\left(u_{y}^{2}+v_{x}^{2}\right)+\left[1+(\beta+\gamma)^{2}\right] u_{x} v_{y}-\left(\alpha^{2}+\gamma^{2}\right) u_{y} v_{x}}{L} .
$$

The Weyl form is obtained from $\Theta_{C}$ by omitting the final term, i.e., setting $R=0$. The Carathéodory nondegeneracy condion is that the $4 \times 4$ matrix

$$
M=\left(\begin{array}{cccc}
1 & 0 & 0 & \beta+\gamma-R \\
0 & \alpha & R-\gamma & 0 \\
0 & R-\gamma & \alpha & 0 \\
\beta+\gamma-R & 0 & 0 & 1
\end{array}\right)
$$

be nonsingular:

$$
\left[\alpha^{2}-(R-\gamma)^{2}\right]\left[1-(\beta+\gamma-R)^{2}\right] \neq 0,
$$


which, in view of (4.31), depends on the particular values of the first order derivatives of $u$ and $v$. The Weyl nondegeneracy condition is that the matrix $M^{\prime}$ obtained from (4.32) by setting $R=0$ is nonsingular, which holds provided $\alpha \neq \pm \gamma$ and $\beta+\gamma \neq \pm 1$. Again, both nondegeneracy conditions depend on the particular null Lagrangian included in (4.28), and so the degeneracy cannot be ascertained from the Euler-Lagrange equations (4.27) themselves.

The Legendre-Hadamard condition, on the other hand, is simple and conclusive. The symbol of the variational problem is the quadratic biform

$$
Q[\xi, \eta ; \lambda, \mu]=\lambda^{2} \xi^{2}+\alpha \lambda^{2} \eta^{2}+\beta \lambda \mu \xi \eta+\alpha \mu^{2} \xi^{2}+\mu^{2} \eta^{2}
$$

be positive definite, which requires $\alpha>0,|\beta|<\alpha+1$, cf. [41].

Returning to the general discussion of first order Lagrangians, the next step of the equivalence method would be to place the (augmented) Hessian tensor into a canonical form by use of the group action (4.5). Concentrating on the symmetric Hessian (or symbol), we see that this step requires a complete determination of canonical forms for biquadratic biforms, which, as remarked above, has only been done in the cases $p=1$ (mechanics), $q=1$ (scalar field theory), and $p=q=2$. Therefore, the analysis of the general problem requires a much more thorough understanding of the algebraic structure of biforms than is available so far. Note, though, that, in contrast to the two scalar cases, there will be nontrivial moduli associated with the biforms, which consequently introduce additional nontrivial complications in the procedure. For instance, in the stongly elliptic case when $p=q=2$, the canonical moduli $\alpha, \beta$ will, in general, depend on $\left(x, u^{(1)}\right)$, and some form of genericity conditions will be required. Even worse, such equivalence problems will not in general be of "first order constant type", [18; p.37]. Although Cartan has indicated how to continue with the normalization procedure, such problems are notoriously difficult to handle, as remarked on by Gardner, [18; Lecture 4], and Kamran, [27]. In the truly anisotropic cases, $\alpha \pm \beta \neq 1$, there is only a discrete stabilizer group for the canonical form, and so normalizing the symmetric Hessian will prescribe all the group parameters $A, J$. On the other hand, the isotropic cases $\alpha \pm \beta=1$ add an extra twist since there is a residual $\mathrm{SO}(2)$ symmetry for such Lagrangians which can be used to (possibly) normalize the skew Hessian. The isotropy or anisotropy can vary from point to point. I have not yet tried to proceed further with these calculations.

\section{Representation Theory.}

In preparation for a discussion of the higher order theory, we need to collect together some basic facts about the representation theory of the general linear group $\mathrm{GL}(V)$ of a (real) vector space $V$. We refer to Weyl, [51], for the details. The distinct irreducible representation modules of $\mathrm{GL}(V)$ are denoted by $L^{\lambda} V$, called Weyl modules, and indexed by Young diagrams $\lambda=\left(\lambda_{1}, \ldots, \lambda_{k}\right)$, where $\lambda_{1} \geq \lambda_{2} \geq \cdots \geq \lambda_{k}>0$ are positive integers, and $k \leq \operatorname{dim} V$. (If $k>\operatorname{dim} V$, then $L^{\lambda} V=\{0\}$.) Let $|\lambda|=\lambda_{1}+\cdots+\lambda_{k}$ denote the size of the diagram. Visually, Young diagrams are viewed as arrays of boxes, so $\lambda$ would 
correspond to a diagram consisting of $|\lambda|$ boxes arranged with $\lambda_{1}$ boxes in the first row, $\lambda_{2}$ boxes in the second row, etc. In particular, the one-rowed diagram $\lambda=(r)$ corresponds to the symmetric power $\bigodot^{r} V=L^{(r)} V$, while the single column diagram $\lambda=\left(1^{r}\right)$ corresponds to the exterior power $\wedge^{r} V=L^{\left(1^{r}\right)} V$, which is $\{0\}$ if $r>\operatorname{dim} V$.

An important fact is that any polynomial representation of $\mathrm{GL}(V)$ decomposes into a direct sum of irreducible sub-representations. In particular, the $r^{\text {th }}$ order tensor space $\otimes^{r} V$ decomposes into a direct sum of Weyl modules $L^{\lambda} V$ indexed by Young diagrams $\lambda$ representing all partitions of the integer $r$ into at most $p$ parts; the number of copies of each Weyl module $L^{\lambda} V$ is given by an explicit "hook length formula", [17]. Each summand can be explicitly realized as the image of a suitable Young symmetrizer. More generally, any tensor product of two or more representations of GL $(V)$ will itself decompose into irreducible summands. Indeed, the general Littlewood-Richardson rule, [36], gives an explicit GL( $V)$-invariant decomposition of the tensor product of any two Weyl modules into a direct sum of Weyl modules.

For our purposes, we only need the particular "Pieri formula"

$$
V \otimes \bigodot^{r} V=\bigodot^{r+1} V \oplus L^{(r, 1)} V
$$

in which $(r, 1)$ denotes the Young diagram consisting of $r$ boxes in the first row and one box in the second. We investigate the explicit splitting (5.1) in some detail. Let $\pi_{\odot}, \pi_{\wedge}$ denote the projections of $V \otimes \odot^{r} V$ onto the two summands in (5.1). In particular, given a decomposible tensor $v \otimes \mathbf{w} \in V \otimes \odot^{r} V$, we use the notation $\pi_{\odot}(v \otimes \mathbf{w})=v \odot \mathbf{w}$ and $\pi_{\wedge}(v \otimes \mathbf{w})=v \star \mathbf{w}$ to denote its projections. Note that $\odot$ is the standard symmetric product in $\odot^{*} V$; similarly, we view $\star$ as a sort of bilinear "product" between $V$ and $\odot^{r} V$ with image in $L^{(r, 1)} V$. A basis for the "skew" summand $L^{(r, 1)} V$ is given by $\left\{e_{j} \star e_{I}\right\}$ where $I=\left(i_{1}, \ldots, i_{r}\right)$ is a symmetric multi-index, with $1 \leq i_{\nu} \leq p$, and also $j>\min \left\{i_{\nu}\right\}$. This is verified using the well-known straightening formula

$$
\sum_{k \in K} \widetilde{\imath}_{k} e_{k} \star e_{K \backslash k}=0
$$

where the sum is over all different indices $1 \leq k \leq p$ occurring in the symmetric multiindex $K=\left(k_{1}, \ldots, k_{r+1}\right)$. Here $K \backslash k$ denotes the multi-index of order $r-1$ obtained by deleting the index $k$ from $K$, and the interior summation is only over all the distinct indices $1 \leq k \leq p$ which are in $K$. (For example, if $K=(1,1,1,3)$, then there are only two terms in the interior summation, corresponding to $k=1$ or 3 , with $K \backslash k=(1,1,3)$, or $(1,1,1)$ respectively.) If

$$
B=\sum_{k, I} B^{k, I} e_{k} \otimes e_{I},
$$

is any element of $V \otimes \odot^{r} V$, then the symmetric part of $B$ is the tensor $S=\pi_{\odot}(B)$, with coefficients

$$
S^{K}=\sum_{k \in K} B^{k,(K \backslash k)}, \quad \# K=r+1
$$


which is completely symmetric in the multi-index $K$. For example, if $r=3$, then

$$
S^{111}=B^{1,11}, \quad S^{112}=B^{1,12}+B^{2,11}, \quad S^{123}=B^{1,23}+B^{2,13}+B^{3,12} .
$$

The skew part of $B$ is the tensor $W=\pi_{\wedge}(B)$. Utilizing the straightening law (5.2) to express the resulting tensor in terms of the basis of $L^{(r, 1)} V$, we find that the coefficients of $W$ are

$$
W^{j, I}=B^{j, I}-\frac{\widetilde{\imath}_{j}+1}{\widetilde{\imath}_{k}} B^{k, I_{j}}, \quad \# I=r, \quad j>k=\min \left\{i_{\nu}\right\},
$$

where $I_{j}$ is the multi-index obtained from $I$ by replacing one occurrence of $k$, the minimal index in $I$, by $j$. For example

$$
W^{2,111}=B^{2,111}-\frac{1}{3} B^{1,112}, \quad W^{2,122}=B^{2,122}-3 B^{1,222}, \quad W^{2,134}=B^{2,134}-B^{1,234} .
$$

A tensor $B \in V \otimes \bigodot^{r} V$ is symmetric if and only if its skew component is zero: $\pi_{\wedge}(B)=0$. The following elementary direct characterization of symmetric tensors is of use.

Lemma 13. A tensor $B \in V \otimes \bigodot^{r} V$ is symmetric if and only if

$$
\widetilde{\imath}_{k} B^{j, J}=\widetilde{\imath}_{j} B^{k, K} \quad \text { whenever } \quad(j, J)=(k, K)=I, \quad \# I=r+1 .
$$

Moreover, in this case,

$$
B^{j, J}=\frac{\widetilde{\imath}_{k}}{r+1} S^{I}, \quad(j, J)=I
$$

where $S=\pi_{\odot}(B)$ is the symmetric projection of $B$.

An even more complicated way of constructing representations of the general linear group $\mathrm{GL}(V)$ of a vector space $V$ is through the operation of "plethysm". In its simplest form, consider a Weyl module $W=L^{\lambda} V$. Considering $W$ as a vector space in its own right, we can form Weyl modules $L^{\mu} W$ corresponding to irreducible representations of the group $\mathrm{GL}(W)$. When we restrict to the subgroup corresponding to $\mathrm{GL}(V)$, the plethysm $L^{\mu} W=$ $L^{\mu}\left(L^{\lambda} V\right)$ will decompose into irreducible summands which are the Weyl modules for $V$. Unfortunately, a general combinatorial rule for computing plethysms is unknown. See [36] for a discussion (in the isomophic context of "Schur functions") and [15] for extensive tables. We will, however, require one particular plethysm whose explicit decomposition is known.

Theorem 14. Let $V$ be a vector space. Then we have the symmetric plethysm decomposition

$$
\bigodot^{2}\left(\odot^{r} V\right)=\bigoplus_{\nu} L^{\nu} V
$$

In (5.8), the sum is over all partitions $\nu=\left(\nu_{1}, \ldots, \nu_{k}\right)$ of $2 r=|\nu|$ into even parts: $\nu_{\kappa} \in 2 \mathbb{N}$.

For example, $\bigodot^{2}\left(\bigodot^{2} V\right)=\bigodot^{4} V \oplus L^{(2,2)} V$. 


\section{Equivalence of Higher Order Lagangians.}

We now attempt to apply the Cartan equivalence method for the equivalence problem for higher order Lagrangians. We will be able to normalize enough to deduce analogues of the nondegeneracy conditions and, in the second order case, the Cartan form. However, for Lagrangians of order 3 or more, significant new complications arise, and there does not seem to be any clear way to complete the normalization procedure far enough to deduce a satisfactory invariant Cartan form. Indeed, even though the leading terms can be normalized through the equivalence method, and a very promising generalization of the second order version is apparent - the "semi-invariant" Cartan form - it is not invariant under arbitrary point transformations, and hence will not be a consequence of the Cartan method. This appears to be yet another manifestation of the known difficulties surrounding the construction of Cartan forms for Lagrangians of order three or more.

In the implementation of Cartan's absorption and normalization procedure, we proceed by analogy with the case of mechanics $(p=1)$ discussed in [25]. We first compute the differentials of the lifted coframe elements

$$
\xi^{i}=J_{j}^{i}\left(L^{\bar{p}} d x^{j}+\sum_{\# K=s \leq r-1} B_{\alpha}^{j, K} L^{-s \bar{p}} \theta_{K}^{\alpha}\right), \quad i=1, \ldots, p .
$$

They are of the form

$$
d \xi^{i}=\varphi_{j}^{i} \wedge \xi^{j}+\sum_{\# K \leq r-1} \beta_{\alpha}^{i, K} \wedge \vartheta_{K}^{\alpha}+\tau^{i}
$$

where the essential torsion $\boldsymbol{\tau}$ is computed modulo $\mathcal{I}^{(r-1)}$, as indicated by the $\equiv \operatorname{sign}{ }^{\dagger}$ :

$$
\begin{aligned}
\tau^{i} & \equiv J_{j}^{i}\left\{\sum_{\# K=r} \bar{p} L^{\bar{p}-1} \frac{\partial L}{\partial u_{K}^{\alpha}} \theta_{K}^{\alpha} \wedge d x^{j}-\sum_{\# J=r-1} B_{\alpha}^{j, J} L^{1-r \bar{p}} d u_{J, k}^{\alpha} \wedge d x^{k}\right\} \\
& \equiv \bar{A}_{\beta}^{\alpha} J_{j}^{i} J_{K}^{L} \bar{J}_{n}^{m} \sum_{\# K=r}\left\{\bar{p} L^{\bar{p}-1} \frac{\partial L}{\partial u_{K}^{\alpha}} \delta_{m}^{j}-\sum_{k \in K} B_{\alpha}^{j,(K \backslash k)} \delta_{m}^{k}\right\} \vartheta_{L}^{\beta} \wedge \xi^{n} .
\end{aligned}
$$

As in the first order case, the Maurer-Cartan forms $\varphi_{j}^{i}$ are restricted only by the requirement that they be trace-free: $\varphi_{i}^{i}=0$. Therefore, in (6.2), we can absorb all the torsion

$\dagger$ Here, due to a scarcity of suitable letters, we are forced to occasionally use $J$ and $L$ to denote multi-indices, despite the fact that $J$ also denotes some of the group parameters (not to mention the jet bundles!) and $L$ the Lagrangian. However, as the multi-indices only occur as super- and subscripts, it shouldn't be too confusing to distinguish between the two uses in what follows. 
coefficients in $\tau^{i}$ except for the trace. Using the identities (3.5), we find this to be

$$
\begin{gathered}
\bar{A}_{\beta}^{\alpha} J_{j}^{i} J_{K}^{L} \bar{J}_{i}^{m} \sum_{\# K=r}\left\{\bar{p} L^{\bar{p}-1} \frac{\partial L}{\partial u_{K}^{\alpha}} \delta_{m}^{j}-\sum_{k \in K} B_{\alpha}^{j,(K \backslash k)} \delta_{m}^{k}\right\} \\
=\bar{A}_{\beta}^{\alpha} J_{K}^{L} \sum_{\# K=r}\left\{L^{r \bar{p}-1} \frac{\partial L}{\partial u_{K}^{\alpha}}-S_{\alpha}^{K}\right\}
\end{gathered}
$$

where $S^{(r)}=\left(\pi_{\odot} \otimes \mathbb{1}\right) B^{(r)} \in \odot^{r+1} X \otimes U$ is the symmetric component of the tensor $B^{(r)} \in$ $\left(X \otimes \odot^{r} X\right) \otimes U$ based on the Pieri decomposition (5.1), and given explicitly in (5.4). The torsion terms $(6.4)$ can be normalized to 0 by setting

$$
S_{\alpha}^{K}=L^{r \bar{p}-1} \frac{\partial L}{\partial u_{K}^{\alpha}}, \quad \# K=r
$$

which normalizes the symmetric component $S^{(r)}$ of the group tensor $B^{(r)}$.

To normalize the skew component of $B^{(r)}$, we look at the structure equations for the lifted contact coframe elements(2.15). Suppose $\# I=s$, and let $\equiv$ denote equivalence modulo the contact ideal $\mathcal{I}^{(s)}$. We compute

$$
\begin{aligned}
d \vartheta_{I}^{\alpha} & \equiv-\sum_{\# L=s} A_{\beta}^{\alpha} \bar{J}_{I}^{L} L^{-s \bar{p}} \theta_{L, m}^{\beta} \wedge d x^{m} \\
& \equiv-J_{m}^{n} \vartheta_{I, n}^{\alpha} \wedge\left\{\begin{array}{c}
\bar{J}_{i}^{m} \xi^{i}-\sum_{\# K=s \leq r-1} B_{\gamma}^{m, K} L^{-s \bar{p}} \theta_{K}^{\gamma} \\
\equiv
\end{array}\right. \\
& \equiv \xi^{i} \wedge \vartheta_{I, i}^{\alpha}+\sum_{\# K, \# L=r-1} \bar{A}_{\beta}^{\gamma} J_{m}^{n} J_{K}^{L} B_{\gamma}^{m, K} \vartheta_{I, n}^{\alpha} \wedge \vartheta_{L}^{\beta}+\ldots,
\end{aligned}
$$

where we have just indicated the top order contact terms. Now, as we are computing modulo the contact ideal $\mathcal{I}^{(s)}$, the indicated terms will constitute essential torsion provided $r-1>s$, which can certainly be arranged because we are working with a Lagrangian of order $r>1$ by assumption. Therefore, we are allowed to normalize some or all of the torsion terms. In particular, we can set $s=0$, and require that the torsion tensor $\mathbf{T} \in X \otimes \odot^{r-1} X \otimes U$, with components

$$
T_{\beta}^{n, L}=\bar{A}_{\beta}^{\gamma} J_{m}^{n} J_{K}^{L} B_{\gamma}^{m, K},
$$

be symmetric, i.e., $\mathbf{T} \in \odot^{r} X \otimes U$, and hence satisfy condition (5.6). Since the projections onto symmetric and skew components are equivariant under the action of $\mathrm{GL}(X)$, this is equivalent to requiring that $B^{(r)}$ itself be completely symmetric. (Note that we could go even further by requiring that the torsion terms (6.7) all vanish; this is more or less a version of the start of the proof of Bäcklund's Theorem based on the equivalence method. However, this would reduce our structure group too far at the beginning, and, as we remarked earlier, we could never end up with a Cartan form.) Thus, we have proved the following: 
Proposition 15. The condition that $B^{(r)}$ be symmetric is invariant under contact transformations. Consequently, the group normalizations

$$
B_{\alpha}^{j, K}=\frac{\widetilde{\imath}_{j}}{r} L^{r \bar{p}-1} \frac{\partial L}{\partial u_{I}^{\alpha}}, \quad(K, j)=I, \quad \# I=r,
$$

prescribes the leading order normalization of the Cartan form.

The first statement of the proposition can even be checked directly. Indeed, under a change of variables, $B^{(r)}$ gets mapped to $\bar{B}^{(r)}$, where

$$
\bar{B}_{\alpha}^{j, K}=A_{\alpha}^{\beta} \bar{J}_{k}^{j} \bar{J}_{L}^{K} B_{\beta}^{k, L}
$$

which is clearly symmetric if $B^{(r)}$ is.

Now, we've completed the first round of group normalizations. (In the previously treated case of mechanics, we are up to equation $[\mathbf{2 5} ;(2.8)]$.) We now turn to the next loop through the equivalence procedure. We recompute

$$
d \xi^{i}=\varphi_{j}^{i} \wedge \xi^{j}+\sum_{\# J \leq r-2} \beta_{\alpha}^{i, J} \wedge \vartheta_{J}^{\alpha}+\tau^{i} .
$$

There are two components of interest in this essential torsion, which is now computed modulo $\mathcal{I}^{(r-2)}$. First, the $\boldsymbol{\vartheta}^{r-1} \wedge \boldsymbol{\xi}$ terms in $\boldsymbol{\tau}$ will lead directly to nondegeneracy conditions generalizing those discussed in Section 4 for first order Lagrangians. Second, in analogy with [25], the $\boldsymbol{\vartheta}^{r-1} \wedge \boldsymbol{\xi}$ terms in $\boldsymbol{\tau}$ should lead to the required normalizations for the next set of coefficients of the required Cartan form. Since the latter are trickier, we begin with a discussion of the nondegeneracy terms. find

Substituting (6.8) into (6.1), and recomputing the differential $d \xi^{i}$ modulo $\mathcal{I}^{(r-2)}$, we

$$
\tau^{i} \equiv J_{j}^{i}\left\{d L^{\bar{p}} \wedge d x^{j}+d\left(L^{(1-r) \bar{p}} B_{\alpha}^{j, J}\right) \wedge d x^{k}-L^{(2-r) \bar{p}} \widetilde{B}_{\alpha}^{j, M} \theta_{M, k}^{\alpha} \wedge d x^{k}\right\}
$$

where the $B_{\alpha}^{j, J}, \# J=r-1$, are the components of $B^{(r)}$, which have been normalized symmetrically via (6.8), whereas the $\widetilde{B}_{\alpha}^{j, M}, \# M=r-2$, are the as yet unnormalized components of the next lower order tensor $B^{(r-1)}$. The terms involving $\vartheta_{I}^{\beta} \wedge \pi_{K}^{\alpha}$ for $\# I=r-1$, and $\# K=r$ are of the form

$$
\tau^{i}=Y_{\alpha \beta}^{i I K} \vartheta_{I}^{\alpha} \wedge \pi_{K}^{\beta}+\ldots=Z_{\gamma \delta}^{i M N} \theta_{M}^{\gamma} \wedge d u_{N}^{\delta}+\ldots
$$

where, according to $(2.15)$, and the analogous equation for the $\pi_{I}^{\alpha}$ 's,

$$
Y_{\alpha \beta}^{i I K}=L^{(2 r-1) \bar{p}} \bar{A}_{\alpha}^{\gamma} \bar{A}_{\beta}^{\delta} J_{M}^{I} J_{N}^{K} Z_{\gamma \delta}^{i M N}
$$

Substituting for $d x^{j}$ into (6.10) according to (2.14), so

$$
d x^{j} \equiv L^{-\bar{p}} \bar{J}_{i}^{j} \xi^{i}-L^{-r \bar{p}} B_{\alpha}^{j, J} \theta_{J}^{\alpha} \quad \bmod \mathcal{I}^{(r-2)} .
$$


The "augmented Hessian ternsor" $\mathbf{Z}=\left(Z_{\alpha \beta}^{i I K}\right)$ is found to be

$$
\begin{aligned}
Z_{\alpha \beta}^{i I K} & =-\bar{p} L^{(1-r) \bar{p}-1} B_{\alpha}^{i I} \frac{1}{L} \frac{\partial L}{\partial u_{K}^{\beta}}+\frac{\partial\left(B_{\alpha}^{i I} L^{(1-r) \bar{p}}\right)}{\partial u_{K}^{\beta}}+L^{(1-2 r) \bar{p}} \sum_{(m M)=K} B_{\alpha}^{m I} B_{\beta}^{i M} \\
& =\frac{\widetilde{\imath}_{j}}{r} L^{\bar{p}-1} H_{\alpha \beta}^{i I K}+L^{\bar{p}-2} W_{\alpha \beta}^{i I K},
\end{aligned}
$$

where

$$
H_{\alpha \beta}^{J K}=\frac{\partial^{2} L}{\partial u_{J}^{\alpha} \partial u_{K}^{\beta}}, \quad \quad \# J=\# K=r,
$$

are the entries of the usual Hessian matrix $\mathbf{H}$ of $L$ with respect to the $r^{\text {th }}$ order derivatives of $u$, and $\mathbf{W}=\left(W_{\alpha \beta}^{i I K}\right)$, with

$$
W_{\alpha \beta}^{i I K}=\sum_{(m M)=K} \frac{\partial L}{\partial u_{m I}^{\alpha}} \frac{\partial L}{\partial u_{i M}^{\beta}}-\frac{\tilde{\imath}_{j}}{r} \frac{\partial L}{\partial u_{i I}^{\alpha}} \frac{\partial L}{\partial u_{m M}^{\beta}}, \quad \# I=r-1, \# K=r
$$

is the higher order analogue of our first order skew component (4.17). The tensors $\mathbf{Z}$ and W can be viewed as elements of the space $\left(X \otimes \odot^{r-1} X \otimes U^{*}\right) \otimes\left(\odot^{r} X \otimes U^{*}\right)$, which decomposes into irreducible subspaces via repeated application of the Littlewood-Richardson rule. The semi-symmetric Hessian tensor $\mathbf{H}=\left(H_{\alpha \beta}^{J K}\right)$ is an element of the space $\odot^{2}\left(\odot^{r} X \otimes U^{*}\right)$, which decomposes, as in Section 3, into two invariant subspaces: a symmetric component $\odot^{2}\left(\odot^{r} X\right) \otimes \bigodot^{2} U^{*}$ and a skew component $\Lambda^{2}\left(\odot^{r} X\right) \otimes \Lambda^{2} U^{*}$. Each of these in turn, according to the rules of plethysm discussed at the end of Section 5 (in particular Theorem 14) breaks up into further irreducible subspaces; the most important being the component $\odot^{2 r} X \otimes \bigodot^{2} U^{*}$. This is, up to a power of $L$ the symbol of the variational problem,

$$
\Sigma[\xi, \lambda]=H_{\alpha \beta}^{J K} \xi_{J} \xi_{K} \lambda^{\alpha} \lambda^{\beta}=\frac{\partial^{2} L}{\partial u_{J}^{\alpha} \partial u_{K}^{\beta}} \xi_{J} \xi_{K} \lambda^{\alpha} \lambda^{\beta}
$$

which is a biform of bidegree $(2 r, 2)$. As a consequence of these considerations, we deduce the conformal invariance of the symbol under general point (contact) transformations. The higher order Legendre-Hadamard condition would just be the condition that the symbol be positive definite: $\Sigma[\xi, \lambda]>0$ for $\xi \neq 0, \lambda \neq 0$. More generally, the associated nondegeneracy condition will be, as in Section 4, defined by suitably polarizing the symbol. However, the symbol is just one of a bewildering variety of invariant tensors associated with a higher order Lagrangian, whose import remains completely unexplored.

Next we will consider the $\boldsymbol{\vartheta}^{r-1} \wedge \boldsymbol{\xi}$ terms in the essential torsion in the structure equations (6.9). As before, we will be able to normalize the appropriate trace to 0. Such terms arise from two sources in the formula (6.10) for $\tau$ in terms of the base coframe: the $\boldsymbol{\theta}_{r-1} \wedge d \mathbf{x}$ terms and the $d \mathbf{x} \wedge d \mathbf{x}$ terms. Now, expanding (6.10),

$$
\begin{array}{r}
\tau^{i} \equiv J_{j}^{i}\left\{D_{k} L^{\bar{p}} d x^{k} \wedge d x^{j}+\frac{\partial L^{\bar{p}}}{\partial u_{J}^{\alpha}} \theta_{J}^{\alpha} \wedge d x^{j}-D_{k}\left(L^{(1-r) \bar{p}} B_{\alpha}^{j, J}\right) \theta_{J}^{\alpha} \wedge d x^{k}-\right. \\
\left.-L^{(2-r) \bar{p}} \widetilde{B}_{\alpha}^{j, M} \theta_{M, k}^{\alpha} \wedge d x^{k}+\frac{\partial\left(L^{(1-r) \bar{p}} B_{\alpha}^{j, J}\right)}{\partial u_{J}^{\alpha}} \theta_{J}^{\alpha} \wedge \theta_{K}^{\beta}\right\}
\end{array}
$$


where the $B_{\alpha}^{j, J}$ are the components of $B^{(r)}$, which have been normalized symmetrically via (6.8), whereas the $\widetilde{B}_{\alpha}^{j, M}$ are the as yet unnormalized components of the next lower order tensor $B^{(r-1)}$. Next, substituting for $d x^{j}$ according to (6.11), the terms involving $\boldsymbol{\theta}^{r-1} \wedge \boldsymbol{\xi}$, which are the only ones that will lead to $\boldsymbol{\vartheta}^{r-1} \wedge \boldsymbol{\xi}$ terms in $\boldsymbol{\tau}$, are

$$
\begin{aligned}
& L^{-(r+1) \bar{p}} D_{k} L^{\bar{p}}\left\{B_{\alpha}^{k, J} \theta_{J}^{\alpha} \wedge \xi^{i}-J_{j}^{i} \bar{J}_{l}^{k} B_{\alpha}^{j, J} \theta_{J}^{\alpha} \wedge \xi^{l}\right\}+ \\
& \quad+L^{-\bar{p}}\left\{\frac{\partial L^{\bar{p}}}{\partial u_{J}^{\alpha}} \theta_{J}^{\alpha} \wedge \xi^{i}+J_{j}^{i} \bar{J}_{l}^{k}\left[D_{k}\left(L^{(1-r) \bar{p}} B_{\alpha}^{j, J}\right) \theta_{J}^{\alpha}-L^{(2-r) \bar{p}} \widetilde{B}_{\alpha}^{j, M} \theta_{M, k}^{\alpha}\right] \wedge \xi^{l}\right\}
\end{aligned}
$$

We denote the relevant coefficients in the torsion by

$$
\tau^{i}=P_{\alpha, k}^{i, K} \theta_{K}^{\alpha} \wedge \xi^{k}+\ldots=Q_{\beta, k}^{i, M} \vartheta_{M}^{\beta} \wedge \xi^{k}+\ldots
$$

where $\mathbf{P}$ is determined by the previous expression, and, according to (2.14), (2.15),

$$
Q_{\beta, k}^{i, M}=L^{(r-2) \bar{p}} \bar{A}_{\beta}^{\alpha} J_{K}^{M} P_{\alpha, k}^{i, K}
$$

As in the first loop, only the trace

$$
Q_{\beta}^{M}=Q_{\beta, i}^{i, M}
$$

is essential torsion, which we normalize to 0. Clearly, this is the same as requiring the vanishing of the trace

$$
P_{\alpha, i}^{i, K}=(1-p) L^{-(r+1) \bar{p}} B_{\alpha}^{k, J} D_{k} L^{\bar{p}}+L^{-\bar{p}}\left\{-p \frac{\partial L^{\bar{p}}}{\partial u_{J}^{\alpha}}+D_{k}\left(L^{(1-r) \bar{p}} B_{\alpha}^{k, J}\right)-L^{(2-r) \bar{p}} \widetilde{S}_{J}^{\alpha}\right\},
$$

where $\widetilde{S}_{J}^{\alpha}$ are the components of the symmetric component $S^{(r-1)}=\pi_{\odot}\left(B^{(r-1)}\right) \in \odot^{r-2} V$ of $B^{(r-1)}$. Now $(6.15)$ will vanish provided

$$
\begin{aligned}
L^{(2-r) \bar{p}} \widetilde{S}_{J}^{\alpha} & =(p-1) L^{-\bar{p}} D_{k} L^{\bar{p}} B_{\alpha}^{k, J}+p \frac{\partial L^{\bar{p}}}{\partial u_{J}^{\alpha}}-D_{k}\left(L^{(1-r) \bar{p}} B_{\alpha}^{k, J}\right) \\
& =L^{\bar{p}-1}\left(\frac{\partial L}{\partial u_{J}^{\alpha}}-\frac{\tilde{j}_{k}+1}{r} D_{k}\left[\frac{\partial L}{\partial u_{J, k}^{\alpha}}\right]\right),
\end{aligned}
$$

where we have used the earlier normalization (6.8) of $B^{(r)}$ and (remarkably) cancelled two terms. Equation (6.16) provides the required normalization of the symmetric component of $B^{(r-1)}$. However, it no longer appears to be possible to normalize the "skew" component $\pi_{\wedge}\left(B^{(r-1)}\right)$ of the $(r-1)^{\text {st }}$ order group parameter, and we are unable to continue on, at least in any clear manner, in our normalization and reduction of the general Cartan equivalence problem.

In the particular case of a second order Lagrangian $(r=2)$ there are no skew components to $B^{(r-1)}$, and we have determined an invariant Cartan form. Substituting (6.8), 
(6.16), into the original formula, we find that the Cartan form is explicitly given as the wedge product, $c f .(2.16)$, of the $p$ one-forms

$$
\varpi^{j}=L^{\bar{p}} d x^{j}+L^{\bar{p}-1}\left\{\frac{\delta_{k}^{j}+1}{2}\left[\frac{\partial L}{\partial u_{j k}^{\alpha}} \theta_{k}^{\alpha}-D_{k}\left(\frac{\partial L}{\partial u_{j k}^{\alpha}}\right) \theta^{\alpha}\right]+\frac{1}{L} \frac{\partial L}{\partial u_{j}^{\alpha}} \theta^{\alpha}\right\} .
$$

Example 16. Consider, for simplicity, the case of two independent and one dependent variables, $(p=2, q=1)$, with second order Lagrangian $L\left(x, y, u, u_{x}, u_{y}, u_{x x}, u_{x y}, u_{y y}\right)$. The normalized base coframe elements are, according to the general formula (6.17),

$$
\varpi_{x}=\sqrt{L} d x+\frac{1}{\sqrt{L}} \eta_{x}, \quad \varpi_{y}=\sqrt{L} d y+\frac{1}{\sqrt{L}} \eta_{y}
$$

where

$$
\begin{aligned}
& \eta_{x}=L_{u_{x x}} \theta_{x}+\frac{1}{2} L_{u_{x y}} \theta_{y}+\left(L_{u_{x}}-D_{x} L_{u_{x x}}-\frac{1}{2} D_{y} L_{u_{x y}}\right) \theta, \\
& \eta_{y}=\frac{1}{2} L_{u_{x y}} \theta_{x}+L_{u_{y y}} \theta_{y}+\left(L_{u_{y}}-\frac{1}{2} D_{x} L_{u_{x y}}-D_{y} L_{u_{y y}}\right) \theta .
\end{aligned}
$$

The invariant Cartan form is given by

$$
\Theta_{C}=\xi_{x} \wedge \xi_{y}=L d x \wedge d y+\eta_{x} \wedge d y-\eta_{y} \wedge d x+\frac{1}{L} \eta_{x} \wedge \eta_{y}
$$

where $\eta_{x}, \eta_{y}$ are the linear combinations of contact forms given in (6.19). As the reader can check, this form has the requisite properties for a Cartan form, including the encoding of the Euler-Lagrange equations in the form (3.13). Moreover, our derivation proves that this form is invariant under general contact transformations, and thus serves as the direct analogue of the Carathéodory form for second order Lagrangians. Note particularly that, even though we are dealing with a scalar field theory, we are forced to include terms of degree two in the contact forms in order to maintain the invariance of the Cartan form. This form is not the same as the (non-invariant) Cartan form proposed by Krupka and Štěpánková, [35].

The coefficients appearing in our second order Cartan form (6.17) look slightly unusual. Nevertheless, they have a very natural interpretation in terms of the symmetric algebra developed in Section 5, which reveals what one would hope to be the general analogue of the higher order mechanical Cartan form, [25]. To present this, we need to recall a few more facts from symmetric algebra, $[\mathbf{1 6}]$. If $V^{*}$ denotes the dual vector space to a vector space $V$, then its symmetric algebra $\bigodot^{*} V^{*}$ is naturally dual to that of $V$. However, in contrast to the simpler exterior algebra, the natural GL( $V)$-invariant pairing between the two symmetric algebras is complicated by the appearance of binomial coefficients. Let $\left\{e_{1}, \ldots, e_{p}\right\}\left\{\omega^{1}, \ldots, \omega^{p}\right\}$ be dual bases of $V$ and $V^{*}$ respectively, so that the pairing between $V$ and $V^{*}$ has the form $\left\langle\omega^{i} ; e_{j}\right\rangle=\delta_{j}^{i}$. There is an associated basis $\omega^{I}$ of the symmetric algebra, and we have the induced pairing

$$
\left\langle\omega^{I} ; e_{J}\right\rangle=\frac{I !}{r !} \delta_{J}^{I}, \quad \# I=r
$$


between elements of $\odot^{r} V^{*}$ and $\odot^{r} V$. The binomial coefficients in (6.21) are required to ensure that the pairing remain invariant under the action of $\operatorname{GL}(V)$. We define the interior product $\alpha\lrcorner \psi$ between $\alpha \in \bigodot^{s} V, \psi \in \bigodot^{r} V^{*}, r>s$, to be that element of $\bigodot^{r-s} V^{*}$ such that

$$
\langle\alpha\lrcorner \psi ; \beta\rangle=\langle\psi ; \alpha \odot \beta\rangle,
$$

for all $\beta \in \odot^{r-s} V$. Therefore, according to (6.21)

$$
e_{J} \downarrow \omega^{I}=\left\{\begin{array}{cc}
\frac{I !}{(I-J) !} \frac{(r-s) !}{r !} \omega^{I-J}, & J<I, \\
0, & \text { otherwise, }
\end{array} \quad \# I=r, \quad \# J=s\right.
$$

We shall define a collection of "tensorial objects and operators" on our space $X \times U$ associated with an $r^{\text {th }}$ order Lagrangian $L\left(x, u^{(r)}\right)$. These will all be, by construction, invariant under general linear fiber-preserving changes of variable $\bar{x}=A x, \bar{u}=B u$, but, unfortunately, will not, in general, be invariant under more complicated coordinate transformations. Define

$$
\mathbf{L}_{k}=\sum_{\# K=k} \frac{\partial L}{\partial u_{K}^{\alpha}} \frac{\partial^{K}}{\partial x^{K}} \otimes d u^{\alpha}, \quad k=0, \ldots, r
$$

which is a section of the "symbol bundle" $\bigodot^{k} T X \otimes T^{*} U$, and

$$
\Phi_{k}=\sum_{\# K=r} \frac{r !}{K !} \theta_{K}^{\alpha} d x^{K} \otimes \frac{\partial}{\partial u^{\alpha}}, \quad k>0
$$

which is a section of the dual bundle $\bigodot^{k} T^{*} X \otimes T U$. Further define the canonical (identity) $(1,1)$-tensor

$$
d \mathbf{x}=\sum_{i=1}^{p} d x^{i} \otimes \frac{\partial}{\partial x^{i}}
$$

which is a section of $T^{*} X \otimes T X$. We make use of the $T^{*} X$-valued differential operator

$$
\mathbf{D}=\sum_{i=1}^{p} d x^{i} \otimes D_{i}
$$

If $F: J^{r} \rightarrow \mathbb{R}$ is a smooth function, then $\mathbf{D} F$ is just its total exterior derivative. Finally define the $(1,1)$-tensor

$$
\Upsilon=\sum_{i=1}^{p} \varpi^{i} \otimes \frac{\partial}{\partial x^{i}}
$$

by the formula

$$
\left.\Upsilon=L^{\bar{p}} d \mathbf{x}+L^{\bar{p}-1} \sum_{j=0}^{r-1} \sum_{k=0}^{r-1-j}\left[\Phi_{j} \odot(-\mathbf{D})^{k}\right]\right\lrcorner \mathbf{L}_{j+k+1}
$$


The "semi-invariant" Cartan form is defined to be the wedge product

$$
\Theta_{I}=\varpi^{1} \wedge \varpi^{2} \wedge \ldots \wedge \varpi^{p}
$$

of the components of $\Upsilon$. In particular, in the case $p=q=1$, all these tensorial objects are scalars; in particular, $L_{k}=\partial L / \partial u_{k}$, where $u_{k}=D_{x}^{k} u$, and $\Phi_{k}=\theta_{k}$ is the corresponding contact form. Thus, (6.29), (6.30), immediately reduce to the well-known formula

$$
\Theta=L d x+\sum_{j=0}^{r-1} \sum_{k=0}^{r-1-j}\left(-D_{x}\right)^{k}\left(\frac{\partial L}{\partial u_{j+k+1}}\right) \theta_{j}
$$

for the Cartan form in higher order mechanics, [44], [25]. The fact that (6.29) is the natural analogue of the one-variable higher order Cartan form is now clear.

Example 17. Consider the case $r=2$. Formula (6.29) becomes

$$
\left.\left.\Upsilon=L^{\bar{p}}\left\{d \mathbf{x}+\frac{1}{L}\left[\left(\Phi_{1}-\Phi_{0} \odot \mathbf{D}\right)\right\lrcorner \mathbf{L}_{2}+\Phi_{0}\right\lrcorner \mathbf{L}_{1}\right]\right\} .
$$

Suppose, for simplicity, $p=2, q=1$. Since $q=1$, the fiber tangent and cotangent space are both one-dimensional, spanned by $\partial / \partial u$ and $d u$ respectively, and can be effectively ignored in the above formulas. With this proviso, the operator (6.27) takes the explicit form $\mathbf{D}=d x \otimes D_{x}+d y \otimes D_{y}$, and the corresponding forms (6.25) are

$$
\Phi_{1}=\theta_{x} \wedge d x+\theta_{y} \wedge d y, \quad \Phi_{0}=\theta,
$$

(both of which should really be tensored with $\partial_{u}$ ). Hence

$$
\Phi_{1}-\Phi_{0} \odot \mathbf{D}=\left(\theta_{x}-\theta D_{x}\right) \wedge d x+\left(\theta_{y}-\theta D_{y}\right) \wedge d y .
$$

Also, from (6.24),

$$
\mathbf{L}_{1}=L_{u_{x}} \partial_{x}+L_{u_{y}} \partial_{y}, \quad \mathbf{L}_{2}=L_{u_{x x}} \partial_{x}^{2}+L_{u_{x y}} \partial_{x} \odot \partial_{y}+L_{u_{y y}} \partial_{y}^{2},
$$

where we abbreviate $\partial_{x}=\partial / \partial x$, etc. (Again, these should really be tensored with $d u$.) Therefore

$$
\begin{aligned}
\left.\Phi_{0}\right\lrcorner \mathbf{L}_{1}= & L_{u_{x}} \theta_{x} \otimes \partial_{x}+L_{u_{y}} \theta_{y} \otimes \partial_{y}, \\
\left.\left(\Phi_{1}-\Phi_{0} \odot \mathbf{D}\right)\right\lrcorner \mathbf{L}_{2}= & \left\{\left(L_{u_{x x}} \theta_{x}-D_{x} L_{u_{x x}} \theta\right)+\frac{1}{2}\left(L_{u_{x y}} \theta_{y}-D_{y} L_{u_{x y}} \theta\right)\right\} \otimes \partial_{x}+ \\
& +\left\{\frac{1}{2}\left(L_{u_{x y}} \theta_{x}-D_{x} L_{u_{x y}} \theta\right)+\left(L_{u_{y y}} \theta_{y}-D_{y} L_{u_{y y}} \theta\right)\right\} \otimes \partial_{y} .
\end{aligned}
$$

Substituting into (6.32), we deduce that (6.28), which is

$$
\Upsilon=\varpi_{x} \otimes \partial_{x}+\varpi_{y} \otimes \partial_{y},
$$

has components given explicitly by (6.18), (6.19), and hence the semi-invariant Cartan form (6.30) agrees with the Cartan form in (6.20). 
Unfortunately, despite its evident similarity with the Cartan for for higher order mechanics, when $r \geq 3$, the semi-invariant Cartan form (6.29), (6.30), is not invariant under arbitrary point transformations. Indeed, the simple one-parameter group of point transformations generated by the vector field $\mathbf{v}_{1}=\left(x^{1}\right)^{2} \partial / \partial x^{1}$, namely the inversions $x^{1} \mapsto x^{1} /\left(1-t x^{1}\right)$, with $t$ the group parameter, (the other independent and dependent variables being left fixed), does not leave $\Theta_{I}$ invariant. In fact, according to a theorem of Terng, [46], the connected component of the pseudogroup of point transformations on a space of dimension at least 2 is generated by the subgroup of linear transformations and the single additional inversional group generated by $\mathbf{v}_{1}$. So if $\Theta_{I}$ were invariant under this group, it would in fact be invariant under all (orientation preserving) point transformations. Indeed, it is the nonlinear changes of independent variable (!) that destroy the invariance of the semi-invariant Cartan form. However, there is some residual invariance; the following result can be proved by a direct computation, simplified by the fact that our tensorial objects used to define $\Theta_{I}$ are, by construction, invariant under linear changes of independent and of dependent variables.

Theorem 18. For $r \leq 2$, the semi-invariant Cartan form $\Theta_{I}$ is the Cartan form derived by the equivalence method, and is invariant under arbitrary point (contact) transformations. For $r \geq 3$, this form is invariant under arbitrary transformations in the fiber variables, and linear transformations in the base variables.

Return to the equivalence procedure one last time. Recall that we are stuck in our normalization process by the skew components of the group tensor $B^{(r-1)}$. Judging from our normalization of the skew component of $B^{(r)}$, one would expect that these could be normalized by looking at the structure equations for the lifted contact coframe elements. However, this approach runs into unexpected difficulties, which we now try to describe. In order to see the problem more clearly, we need to look a bit closer at the form of the contact structure matrix $A$.

Lemma 19. Suppose $\bar{x}=\varphi(x, u), \bar{u}=\psi(x, u), \bar{u}_{1}=\chi\left(x, u^{(1)}\right)$ is a prolonged point transformation on $J^{2}$. Let $J=D \varphi$ denote the total Jacobian matrix of the base transformation (without the determinantal factor), and let $A$ be the $q \times q$ matrix defined by (4.6). Define

$$
F_{\beta i}^{\alpha}=D_{i} A_{\beta}^{\alpha}, \quad G_{\beta i j}^{\alpha k}=\bar{J}_{m}^{k} A_{\beta}^{\alpha} D_{i} J_{j}^{m}=\bar{J}_{m}^{k} A_{\beta}^{\alpha} D_{i} D_{j} \varphi^{m}
$$

Then, if \#K=s,

$$
\Psi^{*}\left(\bar{\theta}_{K}^{\alpha}\right)=\sum_{\# M=s} \bar{J}_{K}^{M}\left\{A_{\beta}^{\alpha} \theta_{M}^{\beta}+\sum_{(J, j)=M} F_{\beta j}^{\alpha} \theta_{J}^{\beta}+\sum_{(I, i, j)=M} G_{\beta i j}^{\alpha k} \theta_{k, I}^{\beta}+\ldots\right\}
$$

Here the first set of terms consists of $s^{\text {th }}$ order contact forms, the second and third consist of $(s-1)^{\text {st }}$ order contact forms, and the omitted terms are the contact forms of orders $s-2$ and lower. 
In this formula, the $F$-terms basically come from the changes in the dependent variables, whereas the $G$-terms (which will eventually cause problems) arise from the second order derivatives of the transformations of the independent variables. Thus, when we do a corresponding lift of the contact forms, the structure group will contain not only parameters corresponding to the F's in this formula, but also the G's; i.e., (2.15) becomes

$$
\vartheta_{K}^{\alpha}=\sum_{\# L=s} \bar{J}_{K}^{M}\left\{A_{\beta}^{\alpha} L^{-s \bar{p}} \theta_{M}^{\beta}+\sum_{(J, j)=M} F_{\beta j}^{\alpha} L^{(1-s) \bar{p}} \theta_{J}^{\beta}+\sum_{(I, i, j)=M} G_{\beta i j}^{\alpha k} L^{(1-s) \bar{p}} \theta_{k, I}^{\beta}+\ldots\right\}
$$

Now, to be more specific, consider the zero ${ }^{\text {th }}$ order lifted contact forms, $\vartheta^{\alpha}=A_{\beta}^{\alpha} \theta^{\beta}$, i.e., we take $s=0$ in (6.35). We compute modulo $\mathcal{I}^{(0)}$ :

$$
\begin{aligned}
d \vartheta^{\alpha} & \equiv-A_{\beta}^{\alpha} d u_{j}^{\beta} \wedge d x^{j} \equiv L^{\bar{p}} J_{j}^{i} d x^{j} \wedge \vartheta_{i}^{\alpha} \\
& \equiv \xi^{i} \wedge \vartheta_{i}^{\alpha}-\sum_{\# J=s \leq r-1} J_{j}^{i} B_{\beta}^{j, J} L^{-s \bar{p}} \theta_{J}^{\beta} \wedge \vartheta_{i}^{\alpha} .
\end{aligned}
$$

We need to replace the unlifted contact forms by the corresponding expressions in terms of the lifted contact forms. The $(r-1)^{\text {st }}$ order terms have already been normalized so as to be symmetric, so we need to analyze the terms involving $(r-2)^{\text {nd }}$ order lifted contact forms. Using Lemma 19, we find that these terms have the form $P_{\beta}^{i, K} \vartheta_{i}^{\alpha} \wedge \vartheta_{K}^{\beta}$, where the tensor $\mathbf{P}$ breaks up into two pieces, $\mathbf{P}=\mathbf{Q}+\mathbf{R}$, with

$$
\begin{aligned}
Q_{\beta}^{i, K} & =J_{j}^{i} \bar{A}_{\beta}^{\gamma} \bar{A}_{\delta}^{\varepsilon} \sum_{\# N=r} \sum_{(M, m)=N} J_{M}^{K} B_{\varepsilon}^{j, N} F_{\gamma m}^{\delta}, \\
R_{\beta}^{i, K} & =J_{j}^{i} \bar{A}_{\beta}^{\gamma} \bar{A}_{\delta}^{\varepsilon} \sum_{\# N=r(M, m, n)=N} \sum_{M, l} J_{\varepsilon}^{K} B^{j, N} G_{\gamma m n}^{\delta l},
\end{aligned}
$$

where $B^{(r)}=\left(B_{\beta}^{i, K}\right)$ is the symmetric normalized tensor that multiplies the $(r-1)^{\text {st }}$ order contact forms in $\xi$, and is determined by (6.8). Now the goal is to normalize these torsion terms, hopefully to make $\mathbf{P}$ completely symmetric in the indices $(i, K)$. As far as the Q component goes, this is certainly possible since permutations of $(i, K)$ are governed by corresponding permutations of $(j, N)$, and we could normalize $\mathbf{Q}$ to be symmetric in $(j, N)$. However, this symmetric normalization doesn't work on $\mathbf{R}$, since the extra summation index $l$ gets in the way. For example, for a third order Lagrangian, these terms would look like

$$
R_{\beta}^{i k}=J_{j}^{i} J_{l}^{k} \bar{A}_{\beta}^{\gamma} \bar{A}_{\delta}^{\varepsilon} B_{\varepsilon}^{j m n} G_{\gamma m n}^{\delta l},
$$

which is clearly not generally symmetric under permutations of $(i, k)$. This appears to be an essential problem, and prevents us from (obviously) normalizing the Cartan form any further than the top order terms and the symmetric components of the next order terms.

The main question, of course, is how to proceed further. Barring the existence of an infinite-dimensional symmetry group, which is not possible for generic Lagrangians, 
the Cartan equivalence method is guaranteed to eventually (perhaps after prolongation) normalize all the group parameters and thereby deduce the required invariant coframe. The wedge product of the appropriate invariant one-forms, as in (2.16), will then produce a candidate for the invariant Cartan form for a general Lagrangian. However, I have been unable to discern precisely where in the reduction and normalization procedure the determination of the skew components of $B^{(r-1)}$, not to mention the remaining group parameters $B^{(s)}, s<r-1$, is effected, nor do I have any ideas, beyond the semi-invariant components, what the required normalizations could possibly be. This is, in my opinon, a fascinating, but difficult, open problem, that must await further research in this area.

Acknowledgements. It is a pleasure to thank Ian Anderson, Mark Gotay, and Niky Kamran for many stimulating discussions on the Cartan form and the Cartan equivalence method. I would also like to thank Ian Anderson for valuable comments on an earlier draft of this paper. 


\section{References}

[1] Anderson, I.M., Aspects of the inverse problem of the calculus of variations, Archivum Mathematicum (Brno) 24 (1988), 181-202.

[2] Anderson, I.M., The Variational Bicomplex, Academic Press, to appear.

[3] Anderson, I.M., Kamran, N., and Olver, P.J., Internal, external and generalized symmetries, Adv. in Math., to appear.

[4] Bäcklund, A.V., Ueber Flachentransformationen, Math. Ann. 9 (1876), 297-320.

[5] Ball, J.M., Convexity conditions and existence theorems in nonlinear elasticity, Arch. Rat. Mech. Anal. 63 (1977), 337-403.

[6] Ball, J.M., Currie, J.C. and Olver, P.J., Null Lagrangians, weak continuity, and variational problems of arbitrary order, J. Func. Anal. 41 (1981), 135-174.

[7] Betounes, D.E., Extension of the classical Cartan form, Phys. Rev. D 29 (1984), 599-606.

[8] Bodnar, A.C., The Cartan equivalence problem for first order Lagrangians in $m$ independent and $N$ dependent variables, M.Sc. Thesis, Univ. of Waterloo, Ontario, Canada, 1986.

[9] Bryant, R.L., On notions of equivalence of variational problems with one independent variable, Contemp. Math. 68 (1987), 65-76.

[10] Carathéodory, C., Über die Variationsrechnung bei mehrfachen Integralen, Acta Sci. Mat. (Szeged) 4 (1929), 193-216.

[11] Cartan, E., Les problèmes d'équivalence, in: Oeuvres Complètes, part. II, vol. 2, Gauthiers-Villars, Paris, 1952, pp. 1311-1334.

[12] Cartan, E., Sur un problème d'équivalence et la théorie des espaces métriques généralisés, in: Oeuvres Complètes, part. III, vol. 2, Gauthiers-Villars, Paris, 1955, pp. 1131-1153.

[13] Dedecker, P., Calcul des variations et topologie algébrique, Mém. Soc. Roy. Sci. Liège 19 (1957), 1-216.

[14] De Donder, T., Théorie Invariantive du Calcul de Variations, Gauthier-Villars, Paris, 1935.

[15] Eğecioğlu, Ö, and Remmel, J.B., Symmetric and antisymmetric outer plethysms of Schur functions, Atomic Data and Nuclear Data Tables 32 (1985), 157-196.

[16] Federer, H., Geometric Measure Theory, Springer-Verlag, New York, 1969.

[17] Frame, J.S., Robinson, G. de B., and Thrall, R.M., The hook graphs of $S_{n}$, Can. J. Math. 6 (1954), 316-324.

[18] Gardner, R.B., The Method of Equivalence and Its Applications, SIAM, Philadelphia, 1989.

[19] Gardner, R.B. and Shadwick, W.F., Equivalence of one dimensional Lagrangian field theories in the plane I, in: Global Differential Geometry and Global Analysis, D. Ferus et. al., eds., Lecture Notes in Math, vol. 1156, Springer-Verlag, New York, 1985, pp. 154-179.

[20] Gel'fand, I.M. and Fomin, S.V., Calculus of Variations, Prentice Hall, Englewood Cliffs, N.J., 1963. 
[21] Gotay, M., A multisymplectic framework for classical field theory and the calculus of variations: I. Covariant Hamiltonian formalism, in: Mechanics, Analysis and Geometry: 200 Years after Lagrange, M. Francaviglia, eds., Elsevier Science Publishers B.V., New York, 1991, pp. 203-235.

[22] Gotay, M., An exterior differential systems approach to the Cartan form, in: Géométrie Symplectique et Physique Mathématique, P. Donato et. al., eds., Birkhäuser, Boston, 1991, to appear.

[23] Griffiths, P.A., Exterior Differential Systems and the Calculus of Variations, Progress in Math. Vol. 25, Birkhäuser, Boston, 1983.

[24] Hadamard, J., Sur une question de calcul des variations, Bull. Soc. Math. France 30 (1902), 253-2562.

[25] Hsu, L., Kamran, N. and Olver, P.J., Equivalence of higher order Lagrangians II. The Cartan form for particle Lagrangians, J. Math. Phys. 30 (1989), 902-906.

[26] Ibragimov, N.H., Transformation Groups Applied to Mathematical Physics, D. Reidel, Boston, 1985.

[27] Kamran, N., Contributions to the study of the equivalence problem of Elie Cartan and its applications to partial and ordinary differential equations, Mém. Cl. Sci. Acad. Roy. Belg. 45 (1989), Fac. 7.

[28] Kamran, N., and Olver, P.J., Equivalence problems for first order Lagrangians on the line, J. Diff. Eq. 80 (1989), 32-78.

[29] Kamran, N., and Olver, P.J., Le problème d'équivalence à une divergence près dans le calcul des variations des intégrales multiples, Comptes Rendus Acad. Sci. (Paris) Série I 308 (1989), 249-252.

[30] Kamran, N. and Olver, P.J., Equivalence of higher order Lagrangians I. Formulation and reduction, J. Math. Pures et Appliquées 70 (1991), 369-391.

[31] Kamran, N. and Olver, P.J., Equivalence of higher order Lagrangians III. New invariant differential equations, Nonlinearity 5 (1992), 601-621.

[32] Kastrup, H.A., Canonical theories of Lagrangian dynamical systems in physics, Phys. Rep. 101 (1983), 1-167.

[33] Klötzler, R., Mehrdimensionale Variationsrechnung, Birkhäuser Verlag, Basel, 1970.

[34] Krupka, D., Lepagean forms in higher order variational theory, in: Proceedings of IUTAM-ISIMM Symposium on Modern Developments in Analytic Mechanics, S.

Benenti, M. Francaviglia and A. Lichnerowicz, eds., Acta Acad. Sci. Taurinensis 117 (Suppl.) (1983) 197-238.

[35] Krupka, D. and Štěpánková, O., On the Hamilton form in second order calculus of variations, in: Proceedings of the Meeting "Geometry and Physics", M.

Modugno, ed., Pitagora Editrice, Bologna, 1983, pp. 85-101.

[36] Littlewood, D.E., The Theory of Group Characters, Oxford Univ. Press, New York, 1958.

[37] Morrey, C.B., Jr., Multiple integrals in the Calculus of Variations, Springer-Verlag, New York, 1966.

[38] Olver, P.J., Conservation laws and null divergences, Math. Proc. Camb. Phil. Soc. 94 (1983), 529-540. 
[39] Olver, P.J., Applications of Lie Groups to Differential Equations, Graduate Texts in Mathematics, vol. 107, Springer-Verlag, New York, 1986.

[40] Olver, P.J., The equivalence problem and canonical forms for quadratic Lagrangians, Adv. Appl. Math. 9 (1988), 226-257.

[41] Olver, P.J., Canonical elastic moduli, J. Elasticity 19 (1988), 189-212.

[42] Olver, P.J., Canonical anisotropic elastic moduli, in: Modern Theory of Anisotropic Elasticity and Applications, J.J. Wu, T.C.T. Ting and D.M. Barnett, eds., SIAM, Philadelphia, 1991, pp. 325-339.

[43] Rund, H., The Hamilton-Jacobi Theory in the Calculus of Variations, D. Van Nostrand Co. Ltd., Princeton, N.J., 1966 .

[44] Shadwick, W.F., The Hamiltonian formulation of regular $r^{\text {th }}$ order Lagrangian field theories, Lett. Math. Phys. 6 (1982), 409-416.

[45] Sivaloganathan, J., The generalised Hamilton-Jacobi inequality and the stability of equilibria in nonlinear elasticity, Arch. Rat. Mech. Anal. 88 (1988), 347-367.

[46] Terng, C.L., Natural vector bundles and natural differential operators, Amer. J. Math. 100 (1978), 775-828.

[47] Terpstra, F.J., Die Darstellung biquadratische Formen als Summen von Quadraten mit Anwendung auf die Variationsrechnung, Math. Ann. 116 (1939), 166-180.

[48] Turnbull, H.W., Gordan's theorem for double binary forms, Proc. Edinburgh Math. Soc. 41 (1922/23), 116-127.

[49] Turnbull, H.W., A geometrical interpretation of the complete system of the double binary (2,2) form V, Proc. Roy. Soc. Edinburgh 44 (1923/24), 23-50.

[50] Weyl, H., Geodesic fields in the calculus of variations for multiple integrals, Ann. Math. 36 (1935), 607-629.

[51] Weyl, H., Classical Groups, Princeton Univ. Press, Princeton, N.J., 1946. 